\title{
The Generally Covariant Locality Principle - A New Paradigm for Local Quantum Field Theory
}

\author{
Romeo Brunetti $^{(1)}$, Klaus Fredenhagen $^{(2)}$ and Rainer Verch $^{(3)}$ \\ (1) Dip. Scienze Fisiche, Univ. Napoli "Federico II", Comp. Univ. Monte Sant'Angelo, \\ Via Cintia, I-80126 Napoli, and I.N.F.N. sez. Napoli, Italy \\ (2) II. Inst. f. Theoretische Physik, Universität Hamburg, \\ Luruper Chaussee 149, D-22761 Hamburg, Germany \\ (3) Inst. f. Theoretische Physik, Universität Göttingen, \\ Bunsenstrasse 9, D-37073 Göttingen, Germany \\ e-mail: brunetti@na.infn.it, fredenha@x4u2.desy.de, \\ verch@theorie.physik. uni-goettingen.de
}

\begin{abstract}
A new approach to the model-independent description of quantum field theories will be introduced in the present work. The main feature of this new approach is to incorporate in a local sense the principle of general covariance of general relativity, thus giving rise to the concept of a locally covariant quantum field theory. Such locally covariant quantum field theories will be described mathematically in terms of covariant functors between the categories, on one side, of globally hyperbolic spacetimes with isometric embeddings as morphisms and, on the other side, of *-algebras with unital injective *-endomorphisms as morphisms. Moreover, locally covariant quantum fields can be described in this framework as natural transformations between certain functors. The usual Haag-Kastler framework of nets of operator-algebras over a fixed spacetime background-manifold, together with covariant automorphic actions of the isometry-group of the background spacetime, can be re-gained from this new approach as a special case. Examples of this new approach are also outlined. In case that a locally covariant quantum field theory obeys the time-slice axiom, one can naturally associate to it certain automorphic actions, called "relative Cauchy-evolutions", which describe the dynamical reaction of the quantum field theory to a local change of spacetime background metrics. The functional derivative of a relative Cauchy-evolution with respect to the spacetime metric is found to be a divergence-free quantity which has, as will be demonstrated in an example, the significance of an energy-momentum tensor for the locally covariant quantum field theory. Furthermore, we discuss the functorial properties of state spaces of locally covariant quantum field theories that entail the validity of the principle of local definiteness.
\end{abstract}

\section{INTRODUCTION}

Quantum field theory incorporates two main principles into quantum physics, locality and covariance. Locality expresses the idea that quantum processes can be localized in space and time [and, at the level of observable quantities, that causally separated processes are exempt from any uncertainty relations restricting their commensurability]. The principle of Poincaré-covariance within special relativity states that there are no preferred Lorentzian coordinates for the description of physical processes, and thereby the concept of an absolute space as an arena for physical phenomena is abandoned. Yet it is still meaningful to speak of events in 
terms of spacetime points as entities of a given, fixed spacetime background in the setting of special relativistic physics.

In general relativity, however, spacetime points lose this a priori meaning. The principle of general covariance forces one to regard spacetime points simultaneously as members of several, locally diffeomorphic spacetimes. It is rather the relations between distinguished events that have a physical interpretation.

This principle should also be observed when quantum field theory in the presence of gravitational fields is discussed. A first approximation to such situations is to consider quantum fields on a given, curved Lorentzian background spacetime where the sources of the gravitational curvature are described classically and independently of the dynamics of the quantum fields in that background. Due to the weakness of gravitational interactions compared to elementary particle interactions, this is expected to be a reasonable approximation which nevertheless has a range of applicability where nontrivial phenomena occur, like particle creation in strong, or rapidly varying, gravitational fields. The most prominent effects of that sort are the Hawking effect [22] and the Fulling-Unruh effect [17, 43].

For quantum field theory on Minkowski spacetime, one demands that quantum fields behave covariantly under Poincaré-transformations, and there are distinguished states, like the vacuum state, or (multi-) particle states tied to the Wigner-type particle concept. Such states are natural reference states which allow it to fix physical quantities in comparison with experiments. In contradistinction to this familiar case, a generic spacetime manifold need not possess any (non-trivial) spacetime symmetries (isometries), and thus there is in general no restrictive concept of covariance for quantum fields propagating on an arbitrary, but fixed curved background spacetime. (A similar problem arises already for quantum fields in flat spacetime coupled to outer classical fields, and most of what follows applies, mutatis mutandis, also to this case.)

This lack of covariance is a source of serious ambiguities in quantum field theory on curved spacetime, such as the lack of a natural candidate of a vacuum state or a Wigner-type particle concept. In turn, this leads to ambiguities in the concrete determination of physical quantities. This problem was observed some time ago by Wald [47] in his discussion of a renormalization prescription for defining the energymomentum tensor of a quantized field on a curved spacetime $M$ with metric tensor $\boldsymbol{g}=g_{\mu \nu}$. For a classical massless Klein-Gordon field $\varphi$, the canonical energymomentum tensor is at $x \in M$

$$
T_{\mu \nu}(x)=\nabla_{\mu} \varphi(x) \nabla_{\nu} \varphi(x)-\frac{1}{2} g_{\mu \nu}(x) \nabla_{\lambda} \varphi(x) \nabla^{\lambda} \varphi(x),
$$

where $\nabla$ denotes the metric-covariant derivative. For a quantum field $\varphi$ pointwise products are ill-defined, and therefore one starts from the point-split expression

$$
T_{\mu \nu}(x, y)=\left(\nabla_{\mu} \varphi(x) \nabla_{\nu} \varphi(y)-\frac{1}{2} g_{\mu \nu}(x, y)\left(g^{\rho \sigma}(x, y) \nabla_{\rho} \varphi(x) \nabla_{\sigma} \varphi(y)\right)\right)
$$

where $g_{\mu \nu}(x, y)$ is the bitensor obtained from $g_{\mu \nu}$ by parallel transport along the geodesic from $x$ to $y$. Since we are eventually interested in the coincidence limit $y \rightarrow x$ we may consider the points as near as needed to get a unique geodesic, hence we do not have any problem of choices.

The idea is now to subtract from $T_{\mu \nu}(x, y)$ a suitable scalar distribution $t_{\mu \nu}(x, y)$ such that the renormalized energy-momentum tensor

$$
T_{\mu \nu}^{\mathrm{ren}}(x)=\lim _{y \rightarrow x}\left(T_{\mu \nu}(x, y)-t_{\mu \nu}(x, y)\right)
$$

may be defined by a well-determined coincidence limit $y \rightarrow x$. Inspired by the similar situation in Minkowski spacetime, a first approach of going about this may 
be taken as follows: Choose a quasi-free Hadamard state $\omega$ as "reference state", and let

$$
t_{\mu \nu}(x, y)=\omega\left(T_{\mu \nu}(x, y)\right) .
$$

Then $T_{\mu \nu}^{\text {ren }}(x)$ exists as a well defined operator valued distribution in all representations induced by arbitrary (other) Hadamard states [7]. The problem of this definition is the non-uniqueness of the chosen reference state: on a generic curved spacetime, there are plenty of quasifree Hadamard states, and none of them is distinguished in the way the vacuum state is on Minkowski spacetime owing to the circumstance that a generic curved spacetime need not admit non-trivial (conformal) isometries. Therefore, choosing different reference states, one gets quite arbitrary modifications of $T_{\mu \nu}^{\mathrm{ren}}(x)$ in the form of added symmetric numerical tensors; hence, little can be said in this approach, for instance, about the back reaction of quantum matter on the gravitational field.

In order to restrict this ambiguity, Wald imposed as a further requirement a principle of locality and covariance which states that the energy-momentum tensor should only locally depend on the spacetime metric; we will outline this condition further below. Starting from this principle he gave a definition of the subtraction term $t_{\mu \nu}$ which depends only on the local metric. By this method he found a covariantly conserved energy momentum tensor, and, as a byproduct, the conformal anomaly showed up, namely, in the case of the conformally covariant Klein-Gordon field it is not possible to find a $t_{\mu \nu}$ such that the resulting energy-momentum tensor is both conserved and traceless. The ambiguity in the definition of the renormalized energy-momentum tensor is now reduced to a local curvature term 47 .

A similar problem occurred in the definition of Wick-polynomials and of renormalized perturbation theory on Lorentzian manifolds. For instance, the definition of the Wick square $: \varphi^{2}: \omega(x)$ given in [7] also takes the form of a coincidence limit

$$
: \varphi^{2}: \omega(x)=\lim _{y \rightarrow x}(\varphi(x) \varphi(y)-\omega(\varphi(x) \varphi(y)))
$$

where $\omega$ is some fixed reference state (again being a quasifree Hadamard state; the limit procedure has to be properly defined, see, e.g. [7]). Again due to the nonunique choice of a reference state, it turns out that chosing instead of $\omega$ a different reference state $\omega^{\prime}$ results in $: \varphi^{2}:_{\omega}(x)$ to $: \varphi^{2}:_{\omega}(x)+f(x)$ with some smooth function $f$. This ambiguity would actually not be very serious at the level of a description of a quantum field theory in terms of operator algebras, but it enters into the definition of time-ordered products of Wick-polynomials from which, in turn, local $S$-matrix functionals are derived in the sense of perturbation theory whose matrix elements may be compared with physical processes modelled by interacting fields on curved spacetime [6]. Furthermore, a more serious ambiguity enters in the course of the process of infinite renormalization of ultraviolet divergencies in defining the timeordered product of Wick-polynomials. There remains a freedom that corresponds to adding certain products of differential operators contracted with Wick-polynomials to the Lagrangean. While one can show [6] that the perturbative classification of interacting scalar field theories on curved spacetimes is independent of that freedom, the predictive power of the local $S$-matrix thus obtained is somewhat limited because the "renormalization constants" now are, in fact, functions depending on the spacetime points. Therefore, it seems most desirable to invoke a suitable locality and covariance principle so as to reduce that ambiguity affecting the $S$-matrix in a similar way as was done by Wald for the case of the energy-momentum tensor. And, in fact, in recent work by Hollands and Wald [24], this task has been attacked successfully. We should like to point out that related ideas concerning the renormalization of physical quantities for quantum fields in flat spacetime coupled to outer electromagnetic fields have been proposed earlier by Dosch and Müller [13]. 
Let us now briefly look at the locality and covariance condition imposed by Wald 447 in order to reduce the ambiguity of the renormalized energy-momentum tensor of the free, massless scalar field. The condition may be formulated as follows. Suppose that one has a prescription for obtaining $T_{\mu \nu}^{\text {ren }}(x)$ on any curved spacetime. Then such a prescription is local and covariant if the following holds: Whenever one has two spacetimes $M$ and $M^{\prime}$ equipped with metrics $\boldsymbol{g}$ and $\boldsymbol{g}^{\prime}$, respectively, and for some (arbitrary) open subset $U$ of $M$ an isometric diffeomorphism $\kappa: U \rightarrow U^{\prime}$ onto an open subset $U^{\prime}$ of $M^{\prime}$ (so that $\kappa_{*} \boldsymbol{g}=\boldsymbol{g}^{\prime}$ ), then it is required that

$$
\alpha_{\kappa}^{\prime}\left(T_{\mu \nu}^{\prime \text { ren }}\left(x^{\prime}\right)\right)=\kappa_{*} T_{\mu \nu}^{\mathrm{ren}}\left(x^{\prime}\right), \quad x^{\prime} \in U^{\prime},
$$

where $\alpha_{\kappa}^{\prime}: \mathcal{A}_{M^{\prime}}\left(U^{\prime}\right) \rightarrow \mathcal{A}_{M}(U)$ is the canonical isomorphism between the local CCR-algebras $\mathcal{A}_{M^{\prime}}\left(U^{\prime}\right)$ of the Klein-Gordon field on $M^{\prime}$ and $\mathcal{A}_{M}(U)$ of the KleinGordon field on $M$ (cf. [10, 47]), and $T_{\mu \nu}^{\text {ren }}$ is the renormalized energy momentum tensor according to the renormalization prescription on $M$, and $T_{\mu \nu}^{\prime \text { ren }}$ that on $M^{\prime}$ according to the same prescription. In other words, the condition demands that $\kappa_{*} T_{\mu \nu}^{\mathrm{ren}}=T_{\mu \nu}^{\prime \text { ren }}$ up to a canonical algebraic isomorphism (strictly speaking, this is only valid at the level of expectation values in Hadamard states).

Two things should be noted. First, the neighbourhood $U$ was arbitrary, and therefore the information entering into the above condition is local (in the sense of being independent of what happens in the surroundings of $U$ or $U^{\prime}$ ). Secondly, the condition makes considerable use of the fact that the quantum theory of the free scalar field can be formulated on every (globally hyperbolic) spacetime and that there is a canonical way of identifying the corresponding quantum field theories on isometrically diffeomorphic subregions of globally different spacetimes by an algebraic isomorphism $\alpha_{\kappa}^{\prime}$. Quantum field theories of that kind respect the dictum of general relativity to regard events (quantum processes) simultaneously as taking place in several, locally isomorphic spacetimes.

The further formalization of this property is the main purpose of the present article. The most general and most efficient mathematical framework for such a discussion is provided by the operator-algebraic approach to quantum field theory which was initiated by Haag and Kastler 21] for quantum field theory on Minkowski spacetime, see also the monographs [19, 1]. In Section 2, we will define a local, generally covariant quantum field theory as a covariant functor between the category of globally hyperbolic (four-dimensional) spacetime manifolds with isometric embeddings as morphisms and the category of $C^{*}$-algebras with invertible endomorphisms as morphisms. This generalizes similar approaches, such as the notion of a local, covariant quantum field recently used in [24], and is very similar to the concept of a covariant field theory over the class of globally hyperbolic manifolds defined in [42]. The latter is a generalization of ideas in [11] where also the setting of categories and functors was used. Our approach seems to have the advantage of generalizing in a natural manner at the same time all these mentioned concepts as well as related ideas on generally covariant quantum field theories which appear e.g. in the famous "Missed opportunities" collection by Dyson 14, or in the works 3, 16, 19]. We will indicate that the theory of a free, scalar Klein-Gordon field on globally hyperbolic spacetimes is an example for our functorial description of a quantum field theory. Moreover, it will turn out that the more common concept of a quantum field theory on a fixed spacetime background described in terms of an isotonous map from bounded open subregions to $C^{*}$-algebras which is covariant when the spacetime possesses isometries (as in the original Haag-Kastler approach on Minkowski-spacetime, as will be indicated below) is actually a consequence of our functorial description.

Section 3 is devoted to a study of the functorial properties of state spaces for locally covariant quantum field theories. A state space will be introduced as a 
contravariant functor between the category of globally hyperbolic spacetimes and the category of dual spaces of $C^{*}$-algebras, with duals of $C^{*}$-algebraic embeddings as morphisms. State spaces will be characterized which have the property that their "local folia" are left invariant under the functorial action of isometric embeddings of spacetime manifolds. These will be seen to obey the principle of local definiteness proposed by Haag, Narnhofer and Stein [20]. We will indicate that the quasifree states of the Klein-Gordon field which fulfill the microlocal spectrum condition [7] or equivalently, the Hadamard condition 32, 29], induce such a state space.

In Section 4 we will demonstrate that for locally covariant quantum field theories obeying the time-slice axiom one can associate a dynamics in the form of automorphic actions, referred to as "relative Cauchy-evolution", which describe the reaction of the quantum field theory on local perturbations of the spacetime metric. We will show that the functional derivative of such relative Cauchy-evolutions with respect to the spacetime-metric is divergence-free. This functional derivative has, in analogy to the case of classical field theory, the significance of an energy-momentum tensor, and in fact we will also show that for the free Klein-Gordon field the functional derivative of the relative Cauchy-evolution agrees with the commutator action of the energy momentum tensor in representations of quasifree Hadamard states.

Finally, in Section 5, as an alternative to the approach by Hollands and Wald [24], we will point out that the construction of local, covariant Wick-polynomials of the free field arises as solution of a simple cohomological problem.

Some technical details appear in an Appendix.

\section{The Generally Covariant Locality Principle}

\subsection{Some Geometrical Preliminaries.}

In the following, we will be concerned with four-dimensional, globally hyperbolic spacetimes, so it is in order to summarize some of their basic properties. For further discussion, see e.g. [23, 46]. We note that the condition of global hyperbolicity doesn't appear to be very restrictive on physical grounds. Its main purpose is to rule out certain causal pathologies.

We denote a spacetime by $(M, \boldsymbol{g})$ where $M$ is a smooth, four-dimensional manifold (smooth meaning here $C^{\infty}$, and Hausdorff, paracompact, and connected) and $\boldsymbol{g}$ is a Lorentzian metric on $M$ (taken to be of signature $(+1,-1,-1,-1)$ ). Also, we always assume that the spacetimes we consider are orientable and time-orientable. The latter means that there exists a $C^{\infty}$-vectorfield $u$ on $M$ which is everywhere timelike, i.e. $\boldsymbol{g}(u, u)>0$. A smooth curve $\gamma: I \rightarrow M, I$ being a connected subset of $\mathbb{R}$, is called causal if $\boldsymbol{g}(\dot{\gamma}, \dot{\gamma}) \geq 0$ where $\dot{\gamma}$ denotes the tangent vector of $\gamma$. Given the global timelike vectorfield $u$ on $M$, one calls a causal curve $\gamma$ future-directed if $\boldsymbol{g}(u, \dot{\gamma})>0$ all along $\gamma$, and analogously one calls $\gamma$ past-directed if $\boldsymbol{g}(u, \dot{\gamma})<0$. This induces a globally consistent notion of time-direction in the spacetime $(M, \boldsymbol{g})$. For any point $x \in M, J^{ \pm}(x)$ denotes the set of all points in $M$ which can be connected to $x$ by a future(+)/past(-)-directed causal curve $\gamma: I \rightarrow M$ so that $x=\gamma(\inf I)$. Two subsets $O_{1}$ and $O_{2}$ in $M$ are called causally separated if they cannot be connected by a causal curve, i.e. if for all $x \in \overline{O_{1}}, J^{ \pm}(x)$ has empty intersection with $\overline{O_{2}}$. By $O^{\perp}$ we denote the causal complement of $O$, i.e. the largest open set in $M$ which is causally separated from $O$.

An orientable and time-orientable spacetime $(M, \boldsymbol{g})$ is called globally hyperbolic if for each pair of points $x, y \in M$ the set $J^{-}(y) \cap J^{+}(x)$ is compact whenever it is non-empty. This property can be shown to be equivalent to the existence of a smooth foliation of $M$ in Cauchy-surfaces, where a smooth hypersurface of $M$ is called a Cauchy-surface if it is intersected exactly once by each inextendible causal curve in $(M, \boldsymbol{g})$ (for precise definition of inextendible causal curve, see the indicated 
references). A particular feature of globally hyperbolic spacetimes is the fact that the Cauchy-problem (inital value problem) for linear hyperbolic wave-equations is well-posed and that such wave-equations possess unique retarded and advanced fundamental solutions on those spacetimes. It should also be observed that global hyperbolicity makes no reference to spacetime isometries.

Of some importance later on will be the concept of isometric embedding. Let $\left(M_{1}, \boldsymbol{g}_{1}\right)$ and $\left(M_{2}, \boldsymbol{g}_{2}\right)$ be two globally hyperbolic spacetimes. A map $\psi: M_{1} \rightarrow M_{2}$ is called an isometric embedding (of $\left(M_{1}, \boldsymbol{g}_{1}\right)$ into $\left(M_{2}, \boldsymbol{g}_{2}\right)$ ) if $\psi$ is a diffeomorphism onto its range $\psi\left(M_{1}\right)$ (i.e. the map $\bar{\psi}: M_{1} \rightarrow \psi\left(M_{1}\right) \subset M_{2}$ is a diffeomorphism) and if $\psi$ is an isometry, that is, $\psi_{*} \boldsymbol{g}_{1}=\boldsymbol{g}_{2}\left\lceil\psi\left(M_{1}\right)\right.$.

\subsection{Quantum Field Theories as Covariant Functors.}

It is a famous saying attributed to E. Nelson that quantum field theory is a functor. This has to do with the map of second quantization, mapping the category of Hilbert-spaces with unitaries as morphisms to that of $\mathrm{C}^{*}$-algebras with unitpreserving $*$-homomorphisms as morphisms. In a similar light, topological quantum field theories have already at an early stage been couched in the framework of categories and functors [2]. Here, we wish to put forward that quantum field theory

is indeed a covariant functor, but in the more fundamental and physical sense of implementing the principles of locality and general covariance, as discussed in the Introduction. As already pointed out, our approach provides a natural generalization both of the usual abstract formulation of quantum field theory in terms of isotonous families of operator algebras indexed by bounded open subregions of a fixed background spacetime, and of other approaches to diffeomorphism-covariant quantum field theory; we will discuss this further below. We first have to define the categories involved in our formulation of locally covariant quantum field theory. (See [30] as general reference on categories and functors.) The two categories we shall use are the following:

Man: This category consists of a class of objects $\operatorname{Obj}(\mathfrak{M a n})$ formed by all fourdimensional, globally hyperbolic spacetimes $(M, \boldsymbol{g})$ which are oriented and time-oriented. Given any two such objects $\left(M_{1}, \boldsymbol{g}_{1}\right)$ and $\left(M_{2}, \boldsymbol{g}_{2}\right)$, the morphisms $\psi \in \operatorname{hom}_{\mathfrak{M a n}}\left(\left(M_{1}, \boldsymbol{g}_{1}\right),\left(M_{2}, \boldsymbol{g}_{2}\right)\right)$ are taken to be the isometric embeddings $\psi:\left(M_{1}, \boldsymbol{g}_{1}\right) \rightarrow\left(M_{2}, \boldsymbol{g}_{2}\right)$ of $\left(M_{1}, \boldsymbol{g}_{1}\right)$ into $\left(M_{2}, \boldsymbol{g}_{2}\right)$ as defined above, but with the additional constraints that

(i) if $\gamma:[a, b] \rightarrow M_{2}$ is any causal curve and $\gamma(a), \gamma(b) \in \psi\left(M_{1}\right)$ then the whole curve must be in the image $\psi\left(M_{1}\right)$, i.e., $\gamma(t) \in \psi\left(M_{1}\right)$ for all $t \in] a, b[$

(ii) the isometric embedding preserves orientation and time-orientation of the embedded spacetime.

The composition rule for any $\psi \in \operatorname{hom}_{\mathfrak{M a n}}\left(\left(M_{1}, \boldsymbol{g}_{1}\right),\left(M_{2}, \boldsymbol{g}_{2}\right)\right)$ and $\psi^{\prime} \in$ $\operatorname{hom}_{\mathfrak{M a n}}\left(\left(M_{2}, \boldsymbol{g}_{2}\right),\left(M_{3}, \boldsymbol{g}_{3}\right)\right)$ is to define its composition $\psi^{\prime} \circ \psi$ as the composition of maps. Hence $\psi^{\prime} \circ \psi:\left(M_{1}, \boldsymbol{g}_{1}\right) \rightarrow\left(M_{3}, \boldsymbol{g}_{3}\right)$ is a well-defined map which is obviously a diffeomorphism onto its range $\psi^{\prime}\left(\psi\left(M_{1}\right)\right)$ and clearly isometric; also the properties $(i)$ and and (ii) are obviously fulfilled, and hence $\psi^{\prime} \circ \psi \in \operatorname{hom}_{\mathfrak{M a n}}\left(\left(M_{1}, \boldsymbol{g}_{1}\right),\left(M_{3}, \boldsymbol{g}_{3}\right)\right)$. The associativity of the composition rule follows from the associativity of the composition of maps. Clearly, each $\operatorname{hom}_{\mathfrak{M a n}}((M, \boldsymbol{g}),(M, \boldsymbol{g}))$ possesses a unit element, given by the identical map $\operatorname{id}_{M}: x \mapsto x, x \in M$.

$\mathfrak{A} \mathfrak{d g}$ : This is the category whose class of objects $\operatorname{Obj}(\mathfrak{A} \mathfrak{l} \mathfrak{g})$ is formed by all $C^{*}$-algebras possessing unit elements, and the morphisms are faithful (injective) unit-preserving $*$-homomorphisms. Given $\alpha \in \operatorname{hom}_{\mathfrak{A} \mathfrak{g}}\left(\mathcal{A}_{1}, \mathcal{A}_{2}\right)$ and 
$\alpha^{\prime} \in \operatorname{hom}_{\mathfrak{A} \mathfrak{g}}\left(\mathcal{A}_{2}, \mathcal{A}_{3}\right)$, the composition $\alpha^{\prime} \circ \alpha$ is again defined as the composition of maps and easily seen to be an element in $\operatorname{hom}_{\mathfrak{A} \mathfrak{g}}\left(\mathcal{A}_{1}, \mathcal{A}_{3}\right)$. The unit element in $\operatorname{hom}_{\mathfrak{A} \mathfrak{g}}(\mathcal{A}, \mathcal{A})$ is for any $A \in \operatorname{Obj}(\mathfrak{A} \mathfrak{g})$ given by the identical map $\operatorname{id}_{\mathcal{A}}: A \mapsto A, A \in \mathcal{A}$.

Remarks. (A) Requirement (i) on the morphisms of $\mathfrak{M a n}$ is introduced in order that the induced and intrinsic causal structures coincide for the embedded spacetime $\psi\left(M_{1}\right) \subset M_{2}$. Aspects of this condition are discussed in [27]. Condition (ii) might, in fact, be relaxed; the resulting structure, allowing also isometric embeddings which reverse spatial- and time-orientation, could accomodate a discussion of PCT-theorems. We hope to report elsewhere on this topic.

(B) Clearly, one may envisage variations on the categories introduced here. Our present choices might have to be changed or supplemented by other structures, depending on the situations considered. For example, instead of choosing for $\operatorname{Obj}(\mathfrak{A} \mathfrak{t} \mathfrak{g})$ the class of $C^{*}$-algebras with unit elements, one could consider $*$-algebras, Borchersalgebras, or von Neumann algebras; we have chosen $C^{*}$-algebras for definiteness. Moreover, one could also allow more general objects than globally hyperbolic spacetimes in $\operatorname{Obj}(\mathfrak{M a n})$, or endow these objects with additional structures, e.g. spinstructures, as in 11, 42. For discussing the locality and covariance structures of observables, however, the present approach appears sufficient.

Now we wish to define the concept of locally covariant quantum field theory.

\subsection{Definition.}

(i) A locally covariant quantum field theory is a covariant functor $\mathscr{A}$ between the two categories $\mathfrak{M a n}$ and $\mathfrak{A} \mathfrak{h}$, i.e., writing $\alpha_{\psi}$ for $\mathscr{A}(\psi)$, in typical diagramatic form:

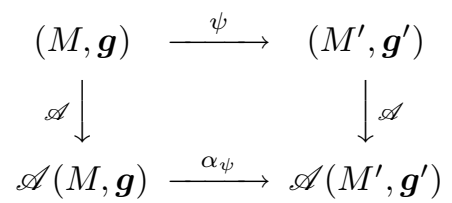

together with the covariance properties

$$
\alpha_{\psi^{\prime}} \circ \alpha_{\psi}=\alpha_{\psi^{\prime} \circ \psi}, \quad \alpha_{\mathrm{id}_{M}}=\operatorname{id}_{\mathscr{A}(M, \boldsymbol{g})},
$$

for all morphisms $\psi \in \operatorname{hom}_{\mathfrak{M a n}}\left(\left(M_{1}, \boldsymbol{g}_{1}\right),\left(M_{2}, \boldsymbol{g}_{2}\right)\right)$, all morphisms $\psi^{\prime} \in$ $\operatorname{hom}_{\mathfrak{M a n}}\left(\left(M_{2}, \boldsymbol{g}_{2}\right),\left(M_{3}, \boldsymbol{g}_{3}\right)\right)$ and all $(M, \boldsymbol{g}) \in \operatorname{Obj}(\mathfrak{M a n})$.

(ii) A locally covariant quantum field theory described by a covariant functor $\mathscr{A}$ is called causal if the following holds: Whenever there are morphisms $\psi_{j} \in$ $\operatorname{hom}_{\mathfrak{M a n}}\left(\left(M_{j}, \boldsymbol{g}_{j}\right),(M, \boldsymbol{g})\right), j=1,2$, so that the sets $\psi_{1}\left(M_{1}\right)$ and $\psi_{2}\left(M_{2}\right)$ are causally separated in $(M, \boldsymbol{g})$, then one has

$$
\left[\alpha_{\psi_{1}}\left(\mathscr{A}\left(M_{1}, \boldsymbol{g}_{1}\right)\right), \alpha_{\psi_{2}}\left(\mathscr{A}\left(M_{2}, \boldsymbol{g}_{2}\right)\right)\right]=\{0\}
$$

where $[\mathcal{A}, \mathcal{B}]=\{A B-B A: A \in \mathcal{A}, B \in \mathcal{B}\}$ for any pair of $C^{*}$-algebras $\mathcal{A}$ and $\mathcal{B}$.

(iii) We say that a locally covariant quantum field theory given by the functor $\mathscr{A}$ obeys the time-slice axiom if

$$
\alpha_{\psi}(\mathscr{A}(M, \boldsymbol{g}))=\mathscr{A}\left(M^{\prime}, \boldsymbol{g}^{\prime}\right)
$$

holds for all $\psi \in \operatorname{hom}_{\mathfrak{M a n}}\left((M, \boldsymbol{g}),\left(M^{\prime}, \boldsymbol{g}^{\prime}\right)\right)$ such that $\psi(M)$ contains a Cauchysurface for $\left(M^{\prime}, \boldsymbol{g}^{\prime}\right)$.

Thus, a locally covariant quantum field theory is an assignment of $C^{*}$-algebras to (all) globally hyperbolic spacetimes so that the algebras are identifyable when the spacetimes are isometric, in the indicated way. Note that we use the term "local" 
in the sense of "geometrically local" in the definition which shouldn't be confused with the meaning of locality in the sense of Einstein causality. Causality properties are further specified in (ii) and (iii) of Def. 2.1. Causality means that the algebras $\alpha_{\psi_{1}}\left(\mathscr{A}\left(M_{1}, \boldsymbol{g}_{1}\right)\right)$ and $\alpha_{\psi_{2}}\left(\mathscr{A}\left(M_{2}, \boldsymbol{g}_{2}\right)\right)$ commute elementwise in the larger algebra $\mathscr{A}(M, \boldsymbol{g})$ when the sub-regions $\psi_{1}\left(M_{1}\right)$ and $\psi_{2}\left(M_{2}\right)$ of $M$ are causally separated (with respect to $\boldsymbol{g}$ ). This property is expected to hold generally for observable quantities which can be localized in certain subregions of spacetimes. The time slice axiom (iii) (also called strong Einstein causality, or existence of a causal dynamical law, cf. [42|) says that an algebra of observables on a globally hyperbolic spacetime is already determined by the algebra of observables localized in any neighbourhood of a Cauchy-surface.

Before continuing, some remarks on related approaches are in order now. In 14], Dyson suggested that one should attempt to generalize the usual Haag-Kastler framework of a general description of quantum field theories on Minkowski spacetime, as we have sketched it in the Introduction, to general spacetime manifolds in such a way that the covariance group $\mathcal{P}_{+}^{\uparrow}$ is replaced by the diffeomorphism group. An approach which is very close in spirit to Dyson's suggestion is due to Bannier [3] who constructed, on $\mathbb{R}^{4}$ as fixed background manifold, a generalized CCR-algebra of the Klein-Gordon field of fixed mass on which the diffeomorphism group acts covariantly by $C^{*}$-automorphisms. Bannier's approach may therefore be regarded as a realization of a functor $\mathscr{A}$ with the above properties but where the domain-category $\mathfrak{M a n}$ is replaced by the subcategory $\mathfrak{M a n}_{\mathbb{R}^{4}}$ whose objects are the globally hyperbolic spacetimes $(M, \boldsymbol{g})$ having $M=\mathbb{R}^{4}$ as spacetime manifolds, and globally hyperbolic sub-spacetimes of those. However, it appears that the restriction to a fixed background manifold like $\mathbb{R}^{4}$ is artificial, and at variance with the principles of general relativity. This is supported by the results in [42] where an approach similar to the one presented here was taken, and which "localizes" Dimock's formulation in [10, 11] where a functorial approach to generally covariant quantum field theory seems to have been proposed for the first time. Like Bannier's work, however, Dimock's proposal lacks the "locality" aspect of general covariance and therefore doesn't completely reveal its strength. It was shown in 42] that the combination of general covariance and (geometrical) locality leads, together with a few other, natural requirements, to a spin-statistics theorem for quantum fields on curved spacetimes.

\subsection{The Klein-Gordon Field.}

The simplest and best studied example of a quantum field theory in curved spacetime is the scalar Klein-Gordon field. As was shown by Dimock [10], its local $C^{*}$-algebras can be constructed easily on each globally hyperbolic spacetime, giving rise to a functor $\mathscr{A}$. To summarize this construction, let $(M, \boldsymbol{g})$ be an object in $\operatorname{Obj}(\mathfrak{M a n})$. Global hyperbolicity entails the well-posedness of the Cauchy-problem for the scalar Klein-Gordon equation on $(M, \boldsymbol{g})$,

$$
\left(\nabla^{a} \nabla_{a}+m^{2}+\xi R\right) \varphi=0
$$

(for smooth, real-valued $\varphi$ ) where $\nabla$ is the covariant derivative of $\boldsymbol{g}, m \geq 0$ and $\xi \geq 0$ are constants, and $R$ is the scalar curvature of $\boldsymbol{g}$. Moreover, it implies that there exist uniquely determined advanced and retarded fundamental solutions of the Klein-Gordon equation, $E^{\mathrm{adv} / \mathrm{ret}}: C_{0}^{\infty}(M, \mathbb{R}) \rightarrow C^{\infty}(M, \mathbb{R})$. Their difference $E=E^{\text {adv }}-E^{\text {ret }}$ is called the causal propagator of the Klein-Gordon equation. Let us denote the range $E\left(C_{0}^{\infty}(M, \mathbb{R})\right)$ by $\mathcal{R}$ (or, sometimes, by $\mathcal{R}(M, \boldsymbol{g})$ for clarity). It can be shown (cf. [10]) that defining

$$
\sigma(E f, E h)=\int_{M} f(E h) d \mu_{\boldsymbol{g}}, \quad f, h \in C_{0}^{\infty}(M, \mathbb{R}),
$$


where $d \mu_{\boldsymbol{g}}$ is the metric-induced volume form on $M$, endowes $\mathcal{R}$ with a symplectic form, and thus $(\mathcal{R}, \sigma)$ is a symplectic space. To this symplectic space one can associate its Weyl-algebra $\mathfrak{W}(\mathcal{R}, \sigma)$, which is generated by a family of unitary elements $W(\varphi), \varphi \in \mathcal{R}$, satisfying the CCR in exponentiated form ("Weyl-relations"),

$$
W(\varphi) W(\tilde{\varphi})=\mathrm{e}^{-i \sigma(\varphi, \tilde{\varphi}) / 2} W(\varphi+\tilde{\varphi}) .
$$

Now, when the constants $m$ and $\xi$ are kept fixed independently of $(M, \boldsymbol{g})$, the symplectic space $(\mathcal{R}, \sigma)$ is entirely determined by $(M, \boldsymbol{g})$, and so is $\mathfrak{W}(\mathcal{R}, \sigma)$. Setting therefore $\mathscr{A}(M, \boldsymbol{g})=\mathfrak{W}\left(\mathcal{R}(M, \boldsymbol{g}), \sigma_{(M, \boldsymbol{g})}\right)$, one obtains a candidate for a covariant functor $\mathscr{A}$ with the properties of Def. 2.1. What remains to be checked is the covariance property. Thus, let $\psi \in \operatorname{hom}_{\mathfrak{M a n}}\left((M, \boldsymbol{g}),\left(M^{\prime}, \boldsymbol{g}^{\prime}\right)\right)$ and let us denote by $E, \mathcal{R}, \sigma$ the propagator, range-space, and symplectic form corresponding to the Klein-Gordon equation (1) on $(M, \boldsymbol{g})$, and by $E^{\prime}, \mathcal{R}^{\prime}, \sigma^{\prime}$ their counterparts with respect to $\left(M^{\prime}, \boldsymbol{g}^{\prime}\right)$. Moreover, we denote by $E^{\psi}, \mathcal{R}^{\psi}, \sigma^{\psi}$ the analogous objects for the spacetime $\left(\psi(M), \psi_{*} \boldsymbol{g}\right)$. It was shown in [10] that, writing $\psi_{*} \varphi=\varphi \circ \psi^{-1}$, there holds $E^{\psi}=\psi_{*} \circ E \circ \psi_{*}{ }^{-1}, \mathcal{R}^{\psi}=\psi_{*} \mathcal{R}$, and $\sigma(E f, E h)=\sigma^{\psi}\left(E^{\psi} \psi_{*} f, E^{\psi} \psi_{*} h\right)=$ $\sigma^{\psi}\left(\psi_{*} E f, \psi_{*} E h\right)$. Thus $\psi_{*}$ furnishes a symplectomorphism between $(\mathcal{R}, \sigma)$ and $\left(\mathcal{R}^{\psi}, \sigma^{\psi}\right)$, and hence, by a standard theorem [5], there is a $C^{*}$-algebraic isomorphism $\tilde{\alpha}_{\psi}: \mathfrak{W}(\mathcal{R}, \sigma) \rightarrow \mathfrak{W}\left(\mathcal{R}^{\psi}, \sigma^{\psi}\right)$ so that

$$
\tilde{\alpha}_{\psi}(W(\varphi))=W^{\psi}\left(\psi_{*}(\varphi)\right), \quad \varphi \in \mathcal{R}
$$

where $W^{\psi}($.$) denote the CCR-generators of \mathfrak{W}\left(\mathcal{R}^{\psi}, \sigma^{\psi}\right)$.

While these observations are already contained in Dimock's work [10], we add another one which is important in the present context: Since $\psi: M \rightarrow \psi(M) \subset M^{\prime}$ is a metric isometry, it holds that $\psi_{*} \boldsymbol{g}=\boldsymbol{g}^{\prime} \uparrow \psi(M)$. And hence the fact that the advanced and retarded fundamental solutions of the Klein-Gordon operator are uniquely determined on a globally hyperbolic spacetime implies that $E^{\psi}=$ $\chi_{\psi(M)} E^{\prime}\left\lceil C_{0}^{\infty}(\psi(M), \mathbb{R})\right.$ where $\chi_{\psi(M)}$ is the characteristic function of $\psi(M)$ and that, moreover, $\mathcal{R}^{\psi}$ can be identified with $E^{\prime}\left(C_{0}^{\infty}(\psi(M), \mathbb{R})\right)$ and $\sigma^{\psi}$ with $\sigma^{\prime} \uparrow \mathcal{R}^{\psi}$. Therefore, the map $T^{\psi}$ which assigns to each element $E f, f \in C_{0}^{\infty}(M, \mathbb{R})$, the element $E^{\prime} \iota_{\psi *} f$ in $\left(\mathcal{R}^{\prime}, \sigma^{\prime}\right)$, is a symplectic map from $\left(\mathcal{R}^{\psi}, \sigma^{\psi}\right)$ into $\left(\mathcal{R}^{\prime}, \sigma^{\prime}\right)$, and thus one obtains a $C^{*}$-algebraic endomorphism $\tilde{\alpha}_{\iota_{\psi}}: \mathfrak{W}\left(\mathcal{R}^{\psi}, \sigma^{\psi}\right) \rightarrow \mathfrak{W}\left(\mathcal{R}^{\prime}, \sigma^{\prime}\right)$ by

$$
\tilde{\alpha}_{\iota \psi}\left(W^{\psi}(\phi)\right)=W^{\prime}\left(T^{\psi} \phi\right), \quad \phi \in \mathcal{R}^{\psi},
$$

where $W^{\prime}($.$) denote the Weyl-generators of \mathfrak{W}\left(\mathcal{R}^{\prime}, \sigma^{\prime}\right)$. Hence, setting $\alpha_{\psi}=\tilde{\alpha}_{\iota_{\psi}}$ 。 $\tilde{\alpha}_{\psi}$, we have a $C^{*}$-algebraic endomorphism $\alpha_{\psi}: \mathscr{A}(M, \boldsymbol{g}) \rightarrow \mathscr{A}\left(M^{\prime}, \boldsymbol{g}^{\prime}\right)$. The covariance property $\alpha_{\psi^{\prime} \circ \psi}=\alpha_{\psi^{\prime}} \circ \alpha_{\psi}$ for $\psi \in \operatorname{hom}_{\mathfrak{M a n}}\left((M, \boldsymbol{g}),\left(M^{\prime}, \boldsymbol{g}^{\prime}\right)\right)$ and $\psi^{\prime} \in$ $\operatorname{hom}_{\mathfrak{M a n}}\left(\left(M^{\prime}, \boldsymbol{g}^{\prime}\right),\left(M^{\prime \prime}, \boldsymbol{g}^{\prime \prime}\right)\right)$ is an easy consequence of the construction of $\alpha_{\psi}$, i.e. of the relations (2) and (3). It was also shown in 10] that causality and time-slice axiom are fulfilled in each $\mathfrak{W}(\mathcal{R}, \sigma)$ in the following sense: (i) If $f, h \in C_{0}^{\infty}(M, \mathbb{R})$ with supp $f \subset(\operatorname{supp} h)^{\perp}$, then $W(E f)$ and $W(E h)$ commute, (ii) if $N$ is an open neighbourhood of a Cauchy-surface $\Sigma$ in $M$, then there is for each $f \in C_{0}^{\infty}(M, \mathbb{R})$ some $h \in C_{0}^{\infty}(N, \mathbb{R})$ with $W(E f)=W(E h)$. We collect these findings in the following

2.2. Theorem. If one defines for each $(M, \boldsymbol{g}) \in \operatorname{Obj}(\mathfrak{M a n})$ the $C^{*}$-algebra $\mathscr{A}(M, \boldsymbol{g})$ as the $C C R$-algebra $\mathfrak{W}\left(\mathcal{R}(M, \boldsymbol{g}), \sigma_{(M, \boldsymbol{g})}\right)$ of the Klein-Gordon equation (1) (with $m, \xi$ fixed for all $(M, \boldsymbol{g}))$, and for each $\psi \in \operatorname{hom}_{\mathfrak{M a n}}\left((M, \boldsymbol{g}),\left(M^{\prime}, \boldsymbol{g}^{\prime}\right)\right)$ the $C^{*}$-algebraic endomorphism $\alpha_{\psi}=\tilde{\alpha}_{\iota_{\psi}} \circ \tilde{\alpha}_{\psi}: \mathscr{A}(M, \boldsymbol{g}) \rightarrow \mathscr{A}\left(M^{\prime}, \boldsymbol{g}^{\prime}\right)$ according to (2) and (3), then one obtains in this way a covariant functor $\mathscr{A}$ with the properties of Def. 2.1. Moreover, this functor is causal and fulfills the time-slice axiom.

In this sense, the free Klein-Gordon field theory is a locally covariant quantum field theory. 


\subsection{Recovering Algebraic Quantum Field Theory.}

Now, we will address the issue of re-gaining the usual setting of algebraic quantum field theory on a fixed globally hyperbolic spacetime from a locally covariant quantum field theory, i.e. from a covariant functor $\mathscr{A}$ with the properties listed above. It may be helpful for readers not too familiar with the algebraic approach to quantum field theory on Minkowski spacetime that we briefly summarize the Haag-Kastler framework [21] so that it becomes apparent in which way the usual description of algebraic quantum field theory is re-gained via Prop. 2.3 from our functorial approach. In the Haag-Kastler framework, the basic structure of the formal description of a quantum system is given by a map $O \mapsto \mathcal{A}(O)$ assigning to each open, bounded region $O$ a $C^{*}$-algebra $\mathcal{A}(O)$. This "local $C^{*}$-algebra" is supposed to contain all the (bounded) observables of the quantum system at hand that can be measured "at times and locations" within the spacetime region $O$; e.g., if the system is described by a hermitean scalar quantum field $\varphi(x)$, then $\mathcal{A}(O)$ may be taken as the operator-algebra generated by all exponentiated field operators $\mathrm{e}^{i \varphi(f)}$ where the test-functions $f$ are supported in $O$, and the smeared field-operators are $\varphi(f)=\int d^{4} x f(x) \varphi(x)$. Hence, one has the condition of isotony, demanding that $\mathcal{A}\left(O_{1}\right) \subset \mathcal{A}(O)$ whenever $O_{1} \subset O$. It is also assumed that the local algebras all contain a common unit element, denoted by 1 . Moreover, as the local algebras contain observables, it is usually demanded that they commute elementwise when their respective localization regions are spacelike separated.

The locality concept being thus formulated, the notion of special relativistic covariance is given the following form: Collecting all local observables in the minimal $C^{*}$-algebra $\mathcal{A}$ containing all local algebras $\mathcal{A}(O)$, 1 there ought to be for each element $L \in \mathcal{P}_{+}^{\uparrow}$ (i.e., the proper, orthochronous Poincaré group) a $C^{*}$-algebra automorphism $\alpha_{L}: \mathcal{A} \rightarrow \mathcal{A}$ so that

$$
\alpha_{L_{1}} \circ \alpha_{L_{2}}=\alpha_{L_{1} \circ L_{2}}, \quad L_{1}, L_{2} \in \mathcal{P}_{+}^{\uparrow},
$$

where $L_{1} \circ L_{2}$ denotes the composition of elements in $\mathcal{P}_{+}^{\uparrow}$.

Let $(M, \boldsymbol{g})$ be an object in $\operatorname{Obj}(\mathfrak{M a n})$. We denote by $\mathcal{K}(M, \boldsymbol{g})$ the set of all subsets in $M$ which are relatively compact and contain with each pair of points $x$ and $y$ also all $\boldsymbol{g}$-causal curves in $M$ connecting $x$ and $y$ (cf. condition (ii) in the definition of $\mathfrak{M a n})$. Given $O \in \mathcal{K}(M, \boldsymbol{g})$, we denote by $\boldsymbol{g}_{O}$ the Lorentzian metric restricted to $O$, so that $\left(O, \boldsymbol{g}_{O}\right)$ (with the induced orientation and time-orientation) is a member of $\operatorname{Obj}(\mathfrak{M a n})$. Then the injection map $\iota_{M, O}:\left(O, \boldsymbol{g}_{O}\right) \rightarrow(M, \boldsymbol{g})$, i.e. the identical map restricted to $O$, is an element in $\operatorname{hom}_{\mathfrak{M a n}}\left(\left(O, \boldsymbol{g}_{O}\right),(M, \boldsymbol{g})\right)$. With this notation, we obtain the following assertion.

2.3. Proposition. Let $\mathscr{A}$ be a covariant functor with the properties stated in Def. 2.1, and define a map $\mathcal{K}(M, \boldsymbol{g}) \ni O \mapsto \mathcal{A}(O) \subset \mathscr{A}(M, \boldsymbol{g})$ by setting

$$
\mathcal{A}(O):=\alpha_{M, O}\left(\mathscr{A}\left(O, \boldsymbol{g}_{O}\right)\right),
$$

having abbreviated $\alpha_{M, O} \equiv \alpha_{\iota_{M, O}}$. Then the following statements hold:

(a) The map fulfills isotony, i.e.

$$
O_{1} \subset O_{2} \Rightarrow \mathcal{A}\left(O_{1}\right) \subset \mathcal{A}\left(O_{2}\right) \text { for all } O_{1}, O_{2} \in \mathcal{K}(M, \boldsymbol{g}) \text {. }
$$

(b) If there exists a group $G$ of isometric diffeomorphisms $\kappa: M \rightarrow M$ (so that $\left.\kappa_{*} \boldsymbol{g}=\boldsymbol{g}\right)$ preserving orientation and time-orientation, then there is a representation $G \ni \kappa \mapsto \tilde{\alpha}_{\kappa}$ of $G$ by $C^{*}$-algebra automorphisms $\tilde{\alpha}_{\kappa}: \mathcal{A} \rightarrow \mathcal{A}$ (where

\footnotetext{
${ }^{1}$ This minimal $C^{*}$-algebra is, as a consequence of the isotony condition, well-defined and in the mathematical terminology called the inductive limit of the family $\{\mathcal{A}(O)\}$ where $O$ ranges over all bounded open subsets of Minkowski spacetime.
} 
$\mathcal{A}$ denotes the minimal $C^{*}$-algebra generated by $\left.\{\mathcal{A}(O): O \in \mathcal{K}(M, \boldsymbol{g})\}\right)$ such that

$$
\tilde{\alpha}_{\kappa}(\mathcal{A}(O))=\mathcal{A}(\kappa(O)), \quad O \in \mathcal{K}(M, \boldsymbol{g}) .
$$

(c) If the theory given by $\mathscr{A}$ is additionally causal, then it holds that

$$
\left[\mathcal{A}\left(O_{1}\right), \mathcal{A}\left(O_{2}\right)\right]=\{0\}
$$

for all $O_{1}, O_{2} \in \mathcal{K}(M, \boldsymbol{g})$ with $O_{1}$ causally separated from $O_{2}$.

(d) Suppose that the theory $\mathscr{A}$ fulfills the time-slice axiom, and let $\Sigma$ be a Cauchysurface in $(M, \boldsymbol{g})$ and let $S \subset \Sigma$ be open and connected. Then for each $O \in$ $\mathcal{K}(M, \boldsymbol{g})$ with $O \supset S$ it holds that

$$
\mathcal{A}(O) \supset \mathcal{A}\left(S^{\perp \perp}\right)
$$

where $S^{\perp \perp}$ is the double causal complement of $S$, and $\mathcal{A}\left(S^{\perp \perp}\right)$ is defined as the smallest $C^{*}$-algebra formed by all $\mathcal{A}\left(O_{1}\right), O_{1} \subset S^{\perp \perp}, O_{1} \in \mathcal{K}(M, \boldsymbol{g})$.

Proof. (a). The proof of this statement is based on the covariance properties of the functor $\mathscr{A}$. To demonstrate that isotony holds, let $O_{1}$ and $O_{2}$ be in $\mathcal{K}(M, \boldsymbol{g})$ with $O_{1} \subset O_{2}$. We denote by $\iota_{2,1}:\left(O_{1}, \boldsymbol{g}_{O_{1}}\right) \rightarrow\left(O_{2}, \boldsymbol{g}_{O_{2}}\right)$ the canonical embedding obtained by restricting the identity map on $O_{2}$ to $O_{1}$, hence $\iota_{2,1} \in \operatorname{hom}_{\mathfrak{M a n}}\left(\left(O_{1}, \boldsymbol{g}_{O_{1}}\right),\left(O_{2}, \boldsymbol{g}_{O_{2}}\right)\right)$. With the notation $\alpha_{\iota_{M, O_{1}}} \equiv \alpha_{M, 1}$, etc., covariance of the functor $\mathscr{A}$ implies $\alpha_{M, 1}=\alpha_{M, 2} \circ \alpha_{2,1}$ and therefore,

$$
\begin{gathered}
\mathcal{A}\left(O_{1}\right)=\alpha_{M, 1}\left(\mathscr{A}\left(O_{1}, \boldsymbol{g}_{O_{1}}\right)\right)=\alpha_{M, 2}\left(\alpha_{2,1}\left(\mathscr{A}\left(O_{1}\right), \boldsymbol{g}_{O_{1}}\right)\right) \\
\subset \alpha_{M, 2}\left(\mathscr{A}\left(O_{2}, \boldsymbol{g}_{O_{2}}\right)\right)=\mathcal{A}\left(O_{2}\right)
\end{gathered}
$$

since $\alpha_{2,1}\left(\mathscr{A}\left(O_{1}, \boldsymbol{g}_{O_{1}}\right)\right) \subset \mathscr{A}\left(O_{2}, \boldsymbol{g}_{O_{2}}\right)$ by the very properties of the functor $\mathscr{A}$.

(b). To prove the second part of the statement, let $\kappa:(M, \boldsymbol{g}) \rightarrow(M, \boldsymbol{g})$ be a diffeomorphism preserving the metric as well as time-orientation and orientation. The functor assigns to it an automorphism $\alpha_{\kappa}: \mathscr{A}(M, \boldsymbol{g}) \rightarrow \mathscr{A}(M, \boldsymbol{g})$. Denoting by $\tilde{\kappa}$ the map $O \rightarrow \kappa(O), x \mapsto \kappa(x)$, there is an associated morphism $\alpha_{\tilde{\kappa}}: \mathscr{A}\left(O, g_{O}\right) \rightarrow$ $\mathscr{A}\left(\kappa(O), \boldsymbol{g}_{\kappa(O)}\right)$. Hence we obtain the following sequence of equations:

$$
\begin{aligned}
\alpha_{\kappa}(\mathcal{A}(O)) & =\alpha_{\kappa} \circ \alpha_{M, O}\left(\mathscr{A}\left(O, \boldsymbol{g}_{O}\right)\right)=\alpha_{\kappa \circ \iota_{M, O}}\left(\mathscr{A}\left(O, \boldsymbol{g}_{O}\right)\right) \\
& =\alpha_{\iota_{M, \kappa(O)} \circ \tilde{\kappa}}\left(\mathscr{A}\left(O, \boldsymbol{g}_{O}\right)\right)=\alpha_{M, \kappa(O)} \circ \alpha_{\tilde{\kappa}}\left(\mathscr{A}\left(O, \boldsymbol{g}_{O}\right)\right) \\
& =\alpha_{M, \kappa(O)}\left(\mathscr{A}\left(\kappa(O), \boldsymbol{g}_{\kappa(O)}\right)\right)=\mathcal{A}(\kappa(O)) .
\end{aligned}
$$

Since $\mathcal{A} \subset \mathscr{A}(M, \boldsymbol{g})$, it follows that defining $\tilde{\alpha}_{\kappa}$ as the restriction of $\alpha_{\kappa}$ to $\mathcal{A}$ yields an automorphism with the required properties. The group representation property is simply a consequence of the covariance properties of the functor yielding $\alpha_{\kappa_{1}} \circ \alpha_{\kappa_{2}}=\alpha_{\kappa_{1} \circ \kappa_{2}}$ for any pair of members $\kappa_{1}, \kappa_{2} \in G$ together with (㺼) which allows us to conclude that $\tilde{\alpha}_{\kappa_{1}} \circ \tilde{\alpha}_{\kappa_{2}}=\tilde{\alpha}_{\kappa_{1} \circ \kappa_{2}}$.

(c). If $O_{1}$ and $O_{2}$ are causally separated members in $\mathcal{K}(M, \boldsymbol{g})$, then one can find a Cauchy-surface $\Sigma$ in $(M, \boldsymbol{g})$ and a pair of disjoint subsets $s_{1}$ and $S_{2}$ of $\Sigma$, both of which are connected and relatively compact, so that $O_{j} \subset S_{j}^{\perp \perp}, j=1,2$. Now $S_{j}^{\perp \perp}$ are causally separated members of $\mathcal{K}(M, \boldsymbol{g})$, and equipped with the appropriate restrictions of $\boldsymbol{g}$ as metrics, they are globally hyperbolic spacetimes in their own right, and naturally embedded into $(M, \boldsymbol{g})$. According to the causally assumption on $\mathscr{A}$, it holds that $\mathcal{A}\left(S_{j}^{\perp \perp}\right)=\alpha_{M, S_{j}^{\perp \perp}}\left(\mathscr{A}\left(S_{j}^{\perp \perp}\right), \boldsymbol{g}_{S_{j}^{\perp \perp}}\right)$ are pairwise commuting subalgebras of $\mathscr{A}(M, \boldsymbol{g})$, and due to isotony, $\mathcal{A}\left(O_{j}\right) \subset \mathcal{A}\left(S_{j}^{\perp \perp}\right)$, so that $\left[\mathcal{A}\left(O_{1}\right), \mathcal{A}\left(O_{2}\right)\right]=\{0\}$.

$(d)$. Consider $S^{\perp \perp}$, equipped with the appropriate restriction of $\boldsymbol{g}$, as a globally hyperbolic spacetime in its own right. Then $S$ is a Cauchy-surface for that spacetime, and $O \cap S^{\perp \perp}$ is an open neighbourhood of the Cauchy-surface $S$. Hence there is an open neighbourhood $N$ of $S$ cointained in $O \cap S^{\perp \perp}$ so that $N$, endowed with 
the restricted metric, is again a globally hyperbolic spacetime. By the time-slice axiom, it follows that $\alpha_{S^{\perp \perp, N}}(\mathscr{A}(N))=\mathscr{A}\left(S^{\perp \perp}\right)$, where we have suppressed the metrics to ease notation. According to the functorial properties of $\mathscr{A}$ it follows that

$$
\mathcal{A}(O) \supset \mathcal{A}(N)=\mathcal{A}\left(S^{\perp \perp}\right) .
$$

This completes the proof.

Thus, one can clearly see that, in the light of Prop. 2.3, the Haag-Kastler framework is a special consequence of our functorial approach.

\subsection{Quantum Fields as Natural Transformations.}

We have just seen how a quantum field theory is defined in terms of a covariant functor. There, an algebra is mapped via the endomorphism $\alpha_{\psi}$ into another algebra, but a priori there are no distiguished elements of the algebras which are mapped onto each other by that transformation. It is however useful to look for such elements and it is actually what motivated the whole approach. Indeed, we shall look at the possibility to define locally covariant fields, and their importance rests on the possibility to construct fields which, in the light of our new principle of locality, depend only locally on the geometry. In a pair of interesting recent papers, Hollands and Wald [24, 25] use this definition to construct Wick-polynomials and time-ordered products of free scalar fields as "local and covariant fields," hence, as objects depending only locally on the metric. In Sec. 5 we shall present our own (but related) derivation of Wick polynomials of a free scalar field, by solving a problem of cohomological nature with a covariance constraint.

In a certain sense our definition gives rise to a locally covariant generalization of the Gårding-Wightman approach to fields as operator-valued distributions. We here do not insist at the beginning on having operators in a Hilbert space but, more abstractly, we consider them as distributions taking values in a topological *-algebra.

The simplest definition may be given as follows: Consider a family $\Phi \equiv\left\{\Phi_{(M, g)}\right\}$, indexed by all spacetimes $(M, \boldsymbol{g}) \in \operatorname{Obj}(\mathfrak{M a n})$, of quantum fields defined as "generalized algebra-valued distributions". That means, there is a family $\{\mathcal{A}(M, \boldsymbol{g})\}$ of topological *-algebras indexed by all spacetimes in $\operatorname{Obj}(\mathfrak{M a n})$, and for each spacetime $(M, \boldsymbol{g}), \Phi_{(M, \boldsymbol{g})}: C_{0}^{\infty}(M) \rightarrow \mathcal{A}(M, \boldsymbol{g})$ is a continuous map (not necessarily linear, this is why we refer to it as a "generalized" distribution). Consider in addition any morphism $\psi \in \operatorname{hom}_{\mathfrak{M a n}}\left(\left(M_{1}, \boldsymbol{g}_{1}\right),\left(M_{2}, \boldsymbol{g}_{2}\right)\right)$. Then we demand that there exists a continuous endomorphism $\alpha_{\psi}: \mathcal{A}\left(M_{1}, \boldsymbol{g}_{1}\right) \rightarrow \mathcal{A}\left(M_{2}, \boldsymbol{g}_{2}\right)$ so that,

$$
\alpha_{\psi}\left(\Phi_{\left(M_{1}, \boldsymbol{g}_{1}\right)}(f)\right)=\Phi_{\left(M_{2}, \boldsymbol{g}_{2}\right)}\left(\psi_{*}(f)\right)
$$

where $f \in C_{0}^{\infty}\left(M_{1}\right)$ is any test function and $\psi_{*}(f)=f \circ \psi^{-1}$ as before. The family $\left\{\Phi_{(M, g)}\right\}$ with these covariance conditions is called a locally covariant quantum field. This simple description has a beautiful functorial translation, as we shall next outline.

We consider again the category $\mathfrak{M a n}$, and introduce the category $\mathfrak{T A} \mathfrak{A}$ consisting of topological *-algebras (with unit elements) as objects, and of continuous *-endomorphisms as morphisms (i.e., $\alpha \in \operatorname{hom}_{\mathfrak{T} \mathfrak{A} \mathfrak{g}}\left(\mathcal{A}_{1}, \mathcal{A}_{2}\right)$ is a morphism of $\mathfrak{T} \mathfrak{A} \mathfrak{g}$ if $\alpha: \mathcal{A}_{1} \rightarrow \mathcal{A}_{2}$ is a continuous, unit-preserving, injective ${ }^{*}$-morphism). In addition, we consider another category $\mathfrak{T}_{\mathfrak{e s t}}$ which is the category containing as objects all possible test-function spaces over $\mathfrak{M a n}$, that is, the objects consist of all spaces $C_{0}^{\infty}(M)$ of smooth, compactly supported test-functions on $M$, for $(M, \boldsymbol{g})$ ranging over the objects of $\mathfrak{M a n}$, and the morphisms are all possible push-forwards $\psi_{*}$ of isometric embeddings $\psi:\left(M_{1}, \boldsymbol{g}_{1}\right) \rightarrow\left(M_{2}, \boldsymbol{g}_{2}\right)$. The action of any push-forward $\psi_{*}$ on an element of a test-function space has been defined above, and it clearly satisfies the requirements for morphisms between test-function spaces. 
Now let a locally covariant quantum field theory $\mathscr{A}$ be defined as a functor in the same manner as in Def. 2.1, but with the category $\mathfrak{T} \mathfrak{A l g}$ in place of the category $\mathfrak{A} \mathfrak{g}$, and again following the convention to denote $\mathscr{A}(\psi)$ by $\alpha_{\psi}$ whenever $\psi$ is any morphism in $\mathfrak{M a n}$. Moreover, let $\mathscr{D}$ be the covariant functor between $\mathfrak{M a n}$ and $\mathfrak{T} e \mathfrak{s t}$ assigning to each $(M, \boldsymbol{g}) \in \operatorname{Obj}(\mathfrak{M a n})$ the test-function space $\mathscr{D}(M, \boldsymbol{g})=C_{0}^{\infty}(M)$, and to each morphism $\psi$ of $\mathfrak{M a n}$ its push-forward: $\mathscr{D}(\psi)=\psi_{*}$. We regard the categories $\mathfrak{T} \mathfrak{e s t}$ and $\mathfrak{T} \mathfrak{A} \mathfrak{g}$ as subcategories of the category of all topological spaces Top, and hence we are led to adopt the following

\subsection{Definition.}

$A$ locally covariant quantum field $\Phi$ is a natural transformation between the functors $\mathscr{D}$ and $\mathscr{A}$, i.e. for any object $(M, \boldsymbol{g})$ in $\mathfrak{M a n}$ there exists a morphism $\Phi_{(M, \boldsymbol{g})}: \mathscr{D}(M, \boldsymbol{g}) \rightarrow \mathscr{A}(M, \boldsymbol{g})$ in $\mathfrak{T} \mathfrak{o p}$ such that for each given morphism $\psi \in \operatorname{hom}_{\mathfrak{M a n}}\left(\left(M_{1}, \boldsymbol{g}_{1}\right),\left(M_{2}, \boldsymbol{g}_{2}\right)\right)$ the following diagram

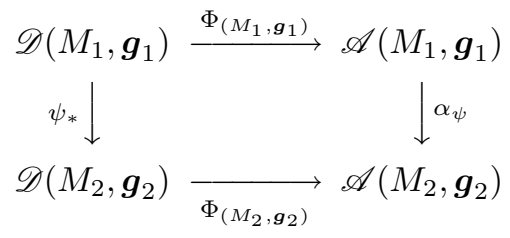

commutes.

The commutativity of the diagram means, explicitly, that

$$
\alpha_{\psi} \circ \Phi_{\left(M_{1}, \boldsymbol{g}_{1}\right)}=\Phi_{\left(M_{2}, \boldsymbol{g}_{2}\right)} \circ \psi_{*}
$$

i.e., the requirement of covariance for fields.

Remarks. (A) This definition may of course be extended; instead of the testfunction spaces $C_{0}^{\infty}(M)$ one may take smooth compactly supported sections of vector bundles, and endomorphisms of such more general test-sections spaces which are suitable pull-backs of vector-bundle endomorphisms. Also, one might include conditions on the wave-front set of the field-operators.

(B) The notion of causality may also be introduced in the obvious manner: One calls a locally covariant quantum field causal if for all $f, h \in \mathscr{D}(M, \boldsymbol{g})$ it holds that $\Phi_{(M, \boldsymbol{g})}(f)$ and $\Phi_{(M, \boldsymbol{g})}(h)$ commute.

(C) One reason for allowing non-linear fields in the definitions of quantum fields as natural transformations is that it can be applied to more general objects. One would be the definition of a locally covariant $S$-matrix, patterned after the definition of a the "local" $S$-amtrix of Epstein and Glaser, see e.g. [6]. At the perturbative level (in the sense of formal power series) this amounts to showing that time-ordered products may be defined in such a way that they become locally covariant fields. Indeed, in a recent paper [25], Hollands and Wald successfully proved the existence of such locally covariant, time-ordered fields. At the non-perturbative level, it might be possible that the constraint of local covariance together with a dynamical generator property (in the spirit of Sec. 4) allows it to fix the phase of the $S$-matrix. We hope to return elsewhere to this issue.

\subsection{Free Scalar Klein-Gordon Field as a Natural Transformation.}

The present subsection serves the purpose of sketching two simple examples for locally covariant quantum fields. The first example is based on the Borchers-Uhlmann algebra which can be associated with each manifold $M$. It assigns to each differentiable manifold $M$ a topological *-algebra $\mathfrak{B}(M)$ that is constructed as follows: Elements in $\mathfrak{B}(M)$ are sequences $\left(f_{n}\right)\left(n \in \mathbb{N}_{0}\right)$ where $f_{0} \in \mathbb{C}$ and $f_{n} \in C_{0}^{\infty}\left(M^{n}\right)$ for $n>0$. Addition and scalar multiplication are defined as usual for sequences 
with values in vector spaces, and the product $\left(f_{n}\right)\left(h_{n}\right)$ in $\mathfrak{B}(M)$ is defined as the sequence $\left(j_{n}\right)$ where

$$
j_{n}\left(x_{1}, \ldots, x_{n}\right)=\sum_{i+j=n} f_{i}\left(x_{1}, \ldots, x_{i}\right) h_{j}\left(x_{i+1}, \ldots, x_{n}\right), \quad\left(x_{1}, \ldots, x_{n}\right) \in M^{n} .
$$

The *-operation is defined via $\left(f_{n}\right)^{*}=\left(\overline{\bar{f}}_{n}\right)$ where $\overline{\bar{f}}_{n}\left(x_{1}, \ldots, x_{n}\right)=\overline{f_{n}\left(x_{n}, \ldots, x_{1}\right)}$, the latter overlining meaning complex conjugation. The unit element is given by $\mathbf{1}=(1,0,0, \ldots)$. The algebra can be equipped with a fairly natural locally convex topology with respect to which it is complete. See [4], 38] (and also [16], [33] in the context of curved spacetime manifolds) for further discussion of the BorchersUhlmann algebra.

Given an endomorphism $\psi \in \operatorname{hom}_{\mathfrak{M a n}}\left(\left(M_{1}, \boldsymbol{g}_{1}\right),\left(M_{2}, \boldsymbol{g}_{2}\right)\right)$, one can lift it to an algebraic endomorphism $\alpha_{\psi}: \mathfrak{B}\left(M_{1}\right) \rightarrow \mathfrak{B}\left(M_{2}\right)$ by setting

$$
\alpha_{\psi}\left(\left(f_{n}\right)\right)=\left(\psi_{*}^{(n)} f_{n}\right)
$$

where $\psi_{*}^{(n)}$ denotes the $n$-fold push-forward, given by $\left(\psi_{*}^{(n)} f_{n}\right)\left(y_{1}, \ldots, y_{n}\right)=$ $f_{n}\left(\psi^{-1}\left(y_{1}\right), \ldots, \psi^{-1}\left(y_{n}\right)\right)$. We thus obtain a covariant functor $\mathscr{A}$ between $\mathfrak{M a n}$ and $\mathfrak{T} \mathfrak{A} \mathfrak{g}$ by setting $\mathscr{A}(M, \boldsymbol{g})=\mathfrak{B}(M)$ and $\mathscr{A}(\psi)=\alpha_{\psi}$ as just defined. A locally covariant quantum field $\Phi$ in the sense of Def. 2.4 may then be obtained by defining for $(M, \boldsymbol{g}) \in \operatorname{Obj}(\mathfrak{M a n})$ and $f \in \mathscr{D}(M, \boldsymbol{g})=C_{0}^{\infty}(M)$,

$$
\Phi_{(M, \boldsymbol{g})}(f)=\left(f_{n}\right)
$$

where $\left(f_{n}\right) \in \mathscr{A}(M, \boldsymbol{g})=\mathfrak{B}(M)$ is the sequence with $f_{1}=f$ and $f_{n}=0$ for all $n \neq 1$. It is straightforward to check that this indeed satisfies all conditions for a natural transformation with respect to the functors $\mathscr{D}$ and $\mathscr{A}$.

The Borchers-Uhlmann algebra, however, carries no dynamical information, which would have to be incorporated by passing to representations, or factorizing by ideals. In this spirit, we introduce as our second example the Klein-Gordon field as a locally covariant field. For $(M, \boldsymbol{g}) \in \operatorname{Obj}(\mathfrak{M a n})$, let $J(M, \boldsymbol{g})$ be the (closed) two-sided ideal in $\mathfrak{B}(M)$ that is generated by all the terms

$$
\left(f_{n}\right)\left(h_{n}\right)-\left(h_{n}\right)\left(f_{n}\right)-\sigma(E f, E h) \mathbf{1}
$$

where the $\left(f_{n}\right)$ and $\left(h_{n}\right)$ in $\mathfrak{B}(M)$ are such that $f_{1}=f, h_{1}=h$, and all other entries in the sequences vanish; $E=E_{(M, g)}$ and $\sigma=\sigma_{(M, g)}$ are the propagator and symplectic form corresponding to the Klein-Gordon equation

$$
\left(\nabla^{\mu} \nabla_{\mu}+\xi R+m^{2}\right) \varphi=0
$$

on $(M, \boldsymbol{g})$ introduced in Subsection 2.3. (Again it is assumed that the constants $\xi$ and $m$ are the same for all $(M, \boldsymbol{g}))$.

Then we introduce a new functor $\mathscr{A}$ between $\mathfrak{M a n}$ and $\mathfrak{T A} \mathfrak{a}$, as follows: We define $\mathscr{A}(M, \boldsymbol{g})=\mathfrak{B}(M) / J(M, \boldsymbol{g})$ and, denoting by [.] : $\mathfrak{B}(M) \rightarrow \mathfrak{B}(M) / J(M, \boldsymbol{g})$ the quotient map, we set for $\psi \in \operatorname{hom}_{\mathfrak{M a n}}\left(\left(M_{1}, \boldsymbol{g}_{1}\right),\left(M_{2}, \boldsymbol{g}_{2}\right)\right)$,

$$
\mathscr{A}(\psi)\left(\left[\left(f_{n}\right)\right]\right) \equiv \alpha_{\psi}\left(\left[\left(f_{n}\right)\right]\right)=\left[\left(\psi_{*}^{(n)} f_{n}\right)\right]
$$

where $\psi_{*}^{(n)}$ is the $n$-fold push-forward of $\psi$ defined above. The required properties of this definition of $\alpha_{\psi}$ to map $J\left(M_{1}, \boldsymbol{g}_{1}\right)$ into $J\left(M_{2}, \boldsymbol{g}_{2}\right)$, and $\alpha_{\psi \circ \psi^{\prime}}=\alpha_{\psi} \circ \alpha_{\psi^{\prime}}$, can be obtained by an argument similar to that in Subsection 2.3 showing that the $\alpha_{\psi}$ defined there have the desired covariance properties.

With respect to this new functor $\mathscr{A}$, we may now define the generally covariant Klein-Gordon field $\Phi$ as a natural transformation according to Def. 2.4 through setting for $(M, \boldsymbol{g}) \in \operatorname{Obj}(M, \boldsymbol{g})$ and $f \in \mathscr{D}(M, \boldsymbol{g})=C_{0}^{\infty}(M)$,

$$
\Phi_{(M, \boldsymbol{g})}(f)=\left[\left(f_{n}\right)\right]
$$


where, as above, $\left(f_{n}\right)$ is the element in $\mathfrak{B}(M)$ with $f_{1}=f$ and $f_{n}=0$ for all $n \neq 1$. Again, the properties of a natural transformation are easily checked for this definition.

Moreover, locally covariant quantum fields $\Phi$ modelling the Klein-Gordon field (5) may be obtained from the functor $\mathscr{A}$ of Subsection 2.3 describing the locally covariant quantum field theory of the Klein-Gordon field at $C^{*}$-algebraic level. We give only a rough sketch of the idea. Let $\mathscr{A}$ be the functor associated with the KleinGordon field in Subsection 2.3. Let $(M, \boldsymbol{g}) \in \operatorname{Obj}(\mathfrak{M a n})$, and let $\pi$ be a Hilbertspace representation of the $C^{*}$-algebra $\mathscr{A}(M, \boldsymbol{g})$ on a representation Hilbert-space $\mathcal{H}$. We assume that there exists a dense subspace $\mathcal{V}$ of $\mathcal{H}$ so that, for each $f \in$ $C_{0}^{\infty}(M, \mathbb{R})$, the field operator

$$
\Phi_{(M, \boldsymbol{g})}(f)=\left.\frac{d}{d s}\right|_{s=0} \pi(W(s E f))
$$

exists as an (essentially) self-adjoint operator on $\mathcal{V}$, where $E$ denotes the propagator and $W($.$) the Weyl-algebra generators associated with the Klein-Gordon field$ on $(M, \boldsymbol{g})$. (The field operators can be extended to all complex-valued testfunctions by requiring complex linearity.) The notation used here already suggests how one may go about in order to try to obtain a locally covariant quantum field in this way. Supposing a quantum field $\Phi_{(M, g)}$ can be defined in this manner for all $(M, \boldsymbol{g}) \in \operatorname{Obj}(\mathfrak{M a n})$ (from representations $\pi$ for each spacetime), and that, for each $\psi \in \operatorname{hom}_{\mathfrak{M a n}}\left((M, \boldsymbol{g}),\left(M^{\prime}, \boldsymbol{g}^{\prime}\right)\right)$, the assignment $\tilde{\alpha}_{\psi}\left(\Phi_{(M, \boldsymbol{g})}(f)\right)=\Phi_{\left(M^{\prime}, \boldsymbol{g}^{\prime}\right)}\left(\psi_{*} f\right)$ extends to a *-algebraic endomorphism $\tilde{\alpha}_{\psi}: \tilde{\mathscr{A}}(M, \boldsymbol{g}) \rightarrow \tilde{\mathscr{A}}\left(M^{\prime}, \boldsymbol{g}^{\prime}\right)$, where $\tilde{\mathscr{A}}(M, \boldsymbol{g})$ denotes the *-algebra formed by all the $\Phi_{(M, g)}(f), f \in C_{0}^{\infty}(M)$, one obtains in this way a locally covariant quantum field $\Phi$ as a natural transformation.

In a similar spirit, Hollands and Wald have constructed Wick-ordered and timeordered products of the free scalar Klein-Gordon field, starting from quasifree Hadamard representations, such that these product-fields are locally covariant fields and are natural transformations in the sense of Def. 2.4 24, 25. We refer to these references for further discussion, and also to Sec. 囵.

\section{States, Representations, and the Principle of Local Definiteness}

\subsection{Functorial Description of a State Space.}

The description of a physical system in terms of operator algebras requires also the concept of states so that expectation values of observables can be calculated. First, suppose that one is given a $C^{*}$-algebra $\mathcal{A}$ with unit element $\mathbf{1}$ modelling the algebra of observables of some physical system. A state is a linear functional $\omega: \mathcal{A} \rightarrow \mathbb{C}$ having the property of being positive, i.e. $\omega\left(A^{*} A\right) \geq 0 \forall A \in \mathcal{A}$, and normalized, i.e. $\omega(\mathbf{1})=1$. Thus, given any hermitean element $A \in \mathcal{A}$, the number $\omega(A)$ is interpreted as an expectation value of the observable $A$ in the state $\omega$.

There is an intimate relation between states on $\mathcal{A}$ and Hilbert-space representations of $\mathcal{A}$. If $\pi$ is a linear $*$-representation of $\mathcal{A}$ by bounded linear operators on some Hilbert-space $\mathcal{H}$, then each positive density matrix $\rho$ with unit trace on $\mathcal{H}$ induces a state $\omega(A)=\operatorname{tr}(\rho \cdot \pi(A)), a \in \mathcal{A}$, on $\mathcal{A}$. There is also a converse of that: For each state $\omega$ on $\mathcal{A}$ there exists a triple $\left(\mathcal{H}_{\omega}, \pi_{\omega}, \Omega_{\omega}\right)$, consisting of a Hilbert-space $\mathcal{H}_{\omega}$, a linear $*$-representation $\pi_{\omega}$ of $\mathcal{A}$ by bounded linear operators on $\mathcal{H}_{\omega}$, and a unit vector $\Omega_{\omega} \in \mathcal{H}_{\omega}$ such that $\omega(A)=\left\langle\Omega_{\omega}, \pi_{\omega}(A) \Omega_{\omega}\right\rangle$ for all $A \in \mathcal{A}$. This triple is called the GNS-representation of $\omega$ (after Gelfand, Naimark and Segal); for its construction, see e.g. [5].

Now suppose that our set of observables arises in terms of a functor $\mathscr{A}$ describing a locally covariant quantum field theory. The question arises what the concept of a state might be in this case. The first, quite natural idea is to say that a 
state is a family $\left\{\omega_{(M, \boldsymbol{g})}:(M, \boldsymbol{g}) \in \operatorname{Obj}(\mathfrak{M a n})\right\}$ indexed by the members in the object-class $\mathfrak{M a n}$ where each $\omega_{(M, \boldsymbol{g})}$ is a state on the $C^{*}$-algebra $\mathscr{A}(M, \boldsymbol{g})$. Usually, however, one is interested in states with particular properties, e.g., one would like to consider states $\omega_{(M, g)}$ fulfilling an appropriate variant of the "microlocal spectrum condition" [7] which can be seen as a replacement for the relativistic spectrum condition for quantum field theories on curved spacetime and which, for free fields, is equivalent to the Hadamard condition (cf. Sec. 2.3, and [32, 34]). One might wonder if, above that, there are families of states $\left\{\omega_{(M, \boldsymbol{g})}:(M, \boldsymbol{g}) \in \operatorname{Obj}(\mathfrak{M a n})\right\}$ that are distinguished by a property which in our framework would correspond to "local diffeomorphism invariance", namely,

$$
\omega_{\left(M^{\prime}, \boldsymbol{g}^{\prime}\right)} \circ \alpha_{\psi}=\omega_{(M, \boldsymbol{g})} \quad \text { on } \mathscr{A}(M, g)
$$

for all $\psi \in \operatorname{hom}_{\mathfrak{M a n}}\left((M, \boldsymbol{g}),\left(M^{\prime}, \boldsymbol{g}^{\prime}\right)\right)$. However, it has been shown in 24] that this invariance property cannot be realized for states of the free scalar field fulfilling the microlocal spectrum condition. Let us briefly sketch an argument showing that the above property will, in general, not be physically realistic. Let us consider two spacetimes $\left(M_{1}, \boldsymbol{g}_{1}\right)$ and $\left(M_{2}, \boldsymbol{g}_{2}\right)$, and assume that $\left(M_{1}, \boldsymbol{g}_{1}\right)$ is just Minkowskispacetime. Moreover, it will be assumed that $\left(M_{2}, \boldsymbol{g}_{2}\right)$ consists of three regions which are themselves globally hyperbolic sub-spacetimes of $\left(M_{2}, \boldsymbol{g}_{2}\right)$ : An "intermediate" region $L_{2}$ lying to the future of a region $N_{2}^{-}$and to the past of a region $N_{2}^{+}$. All these regions are assumed to contain Cauchy-surfaces, and it is also assumed that the regions $N_{2}^{ \pm}$are isometrically diffeomorphic to globally hyperbolic subregions $N_{1}^{ \pm}$of Minkowski spacetime $\left(M_{1}, \boldsymbol{g}_{1}\right)$ which likewise contain Cauchy-surfaces. By $\iota^{ \pm}: N_{1}^{ \pm} \rightarrow N_{2}^{ \pm}$we denote the corresponding isometric diffeomorphisms. We may, for the sake of concreteness, consider a free scalar field (cf. next section), and define the state $\omega_{1}$ on $\mathscr{A}\left(M_{1}, \boldsymbol{g}_{1}\right)$ to be its vacuum state (which fulfillys the microlocal spectrum condition). Then the state $\omega_{2}^{-}=\omega_{1} \circ \alpha_{\iota^{-}}^{-1}$ induces a state on $\mathscr{A}\left(N_{2}^{-}, \boldsymbol{g}_{2, N_{2}^{-}}\right)$and thereby, since the free field obeys the time-slice axiom, it induces a state $\omega_{2}$ on $\mathscr{A}\left(M_{2}, \boldsymbol{g}_{2}\right)$ (which again fulfills the microlocal spectrum condition). Now the state $\omega_{2}$ restricts to a state $\omega_{2}^{+}$on $\mathscr{A}\left(N_{2}^{+}, \boldsymbol{g}_{2, N_{2}^{+}}\right)$. However, if there is non-trivial curvature in the intermediate region $L_{2}$, then the state $\omega_{2}$, which was a vacuum state on the "initial" region $N_{2}^{-}$, will no longer be a vacuum state on the "final" region $N_{2}^{+}$45. The regions $N_{2}^{-}$and $N_{2}^{+}$possess isometric subregions; it is no loss of generality to suppose that there is an isometric diffeomorphism $\psi: N_{2}^{-} \rightarrow N_{2}^{+}$. Then invariance in the above sense of the family of states $\omega_{1}, \omega_{2}, \omega_{2}^{ \pm}$demands that

$$
\omega_{2}^{+} \circ \alpha_{\psi}=\omega_{2}^{-},
$$

but this is not the case ( $\omega_{2}^{-}$is (the restriction of) a vacuum state, $\omega_{2}^{+}$is (the restriction of) a non-vacuum state.) The counterexample is based on a form of "relative Cauchy-evolution", which is worth being studied in greater generality, and this will be the topic of Section 1 .

In view of this negative result one finds oneself confronted with the question if there is a more general concept of "invariance" that can be attributed to families of states $\left\{\omega_{(M, \boldsymbol{g})}:(M, \boldsymbol{g}) \in \operatorname{Obj}(\mathfrak{M a n})\right\}$ for a locally covariant quantum field theory given by a functor $\mathscr{A}$. We will argue that there is a positive answer to that question: The local folia determined by states satisfying the microlocal spectrum condition are good candidates for minimal classes of states which are locally diffeomorphism covariant. To explain this, let us fix some concepts.

Folium of a representation. Let $\mathcal{A}$ be a $C^{*}$-algebra and $\pi: \mathcal{A} \rightarrow B(\mathcal{H})$ a *representation of $\mathcal{A}$ by bounded linear operators on a Hilbert space $\mathcal{H}$. The folium 
of $\pi$, denoted by $\boldsymbol{F}(\pi)$, is the set of all states $\omega^{\prime}$ on $\mathcal{A}$ which can be written as

$$
\omega^{\prime}(A)=\operatorname{tr}(\rho \cdot \pi(A)), \quad A \in \mathscr{A}(M, \boldsymbol{g}) .
$$

In other words, the folium of a representation consists of all density matrix states in that representation.

Local quasi-equivalence and local normality. Let $\mathscr{A}$ be a locally covariant quantum field theory and let, for $(M, \boldsymbol{g})$ fixed, $\omega$ and $\tilde{\omega}$ be two states on $\mathscr{A}$. We will say that these states (or their GNS-representations, denoted by $\pi$ and $\tilde{\pi}$, respectively) are locally quasi-equivalent if for all $O \in \mathcal{K}(M, \boldsymbol{g})$ the relation

$$
\boldsymbol{F}\left(\pi \circ \alpha_{M, O}\right)=\boldsymbol{F}\left(\tilde{\pi} \circ \alpha_{M, O}\right)
$$

is valid, where $\alpha_{M, O}=\alpha_{\iota_{M, O}}$ and $\iota_{M, O}:\left(O, \boldsymbol{g}_{O}\right) \rightarrow(M, \boldsymbol{g})$ is the natural embedding (cf. Prop. 2.3).

Moreover, we say that $\omega$ is locally normal to $\tilde{\omega}$ (or to the corresponding GNSrepresentation $\tilde{\pi}$ ) if

$$
\omega \circ \alpha_{M, O} \in \boldsymbol{F}\left(\tilde{\pi} \circ \alpha_{M, O}\right)
$$

holds for all $O \in \mathcal{K}(M, \boldsymbol{g})$.

Intermediate factoriality. Let $\omega$ be a state on $\mathscr{A}(M, \boldsymbol{g})$, then we define for each $O \in \mathcal{K}(M, \boldsymbol{g})$ the von Neumann algebra $\mathcal{M}_{\omega}(O)=\pi_{\omega}\left(\alpha_{M, O}(\mathscr{A}(M, \boldsymbol{g}))\right)^{\prime \prime}$, the local von Neumann algebra of the region $O$ with respect to the state $\omega$. We say that the state $\omega$ fulfills the condition of intermediate factoriality if for each $O \in \mathcal{K}(M, \boldsymbol{g})$ there exist $O_{1} \in \mathcal{K}(M, \boldsymbol{g})$ and a factorial von Neumann algebra $\mathcal{N}$ acting on the GNS-Hilbert-space $\mathcal{H}_{\omega}$ of $\omega$ so that

$$
\mathcal{M}_{\omega}(O) \subset \mathcal{N} \subset \mathcal{M}_{\omega}\left(O_{1}\right) .
$$

(We recall that a factorial von Neumann algebra $\mathcal{N}$ is a von Neumann algebra so that $\mathcal{N} \cap \mathcal{N}^{\prime}$ contains only multiples of the unit operator.)

It is known that quasifree states of the free scalar field on globally hyperbolic spacetimes which fulfill the microlocal spectrum condition have the property to be locally quasi-equivalent (cf. Subsec. 3.2). Thus, local quasi-equivalence may be expected for states satisfying the microlocal spectrum condition. More generally, local normality can be interpreted as ruling out the possibility of local superselection rules. Also intermediate factoriality is known to hold for states of the free scalar field fulfilling the microlocal spectrum condition on globally hyperbolic spacetimes (cf. again Sec. 3). The condition of intermediate factoriality serves the purpose of eliminating the possible difference between the folium of a representation and the folium of any of its (non-trivial) subrepresentations (see Appendix b)). It can also be motivated as the consequence of a stricter formulation, known a "split property", which is expected to hold for all (also interacting) physically relevant quantum field theories on general grounds (cf. [36, 19, 9]) and is in fact known to hold for states of the free field fulfilling the microlocal spectrum condition in flat and curved spacetimes [8, 40], and for interacting theories in low dimensions [35]. We also note that the property of a state to fulfill the microlocal spectrum condition is a locally covariant property (owing to the covariant behaviour of wavefront sets of distributions under diffeomorphisms [26]) and thus, for a locally covariant quantum field theory it is natural to assume that, if $\omega_{\left(M^{\prime}, \boldsymbol{g}^{\prime}\right)}$ fulfills (any suitable variant of) the microlocal spectrum condition, then so does $\omega_{\left(M^{\prime}, g^{\prime}\right)} \circ \alpha_{\psi}$ for any $\psi \in$ $\operatorname{hom}_{\mathfrak{M a n}}\left((M, \boldsymbol{g}),\left(M^{\prime}, \boldsymbol{g}^{\prime}\right)\right)$. In the case where also the folia of states (i.e., the folia of their GNS-representations) satisfying the microlocal spectrum condition coincide locally, one thus obtains the invariance of local folia under local diffeomorphisms 
for families of states satisfying the microlocal spectrum condition, more precisely, at the level of the GNS-representations of $\omega_{(M, \boldsymbol{g})}$ and $\omega_{\left(M^{\prime}, \boldsymbol{g}^{\prime}\right)}$,

$$
\boldsymbol{F}\left(\pi_{\left(M^{\prime}, \boldsymbol{g}^{\prime}\right)} \circ \alpha_{\psi} \circ \alpha_{M, O}\right)=\boldsymbol{F}\left(\pi_{(M, \boldsymbol{g})} \circ \alpha_{M, O}\right)
$$

holds for all $\psi \in \operatorname{hom}_{\mathfrak{M a n}}\left((M, \boldsymbol{g}),\left(M^{\prime}, \boldsymbol{g}^{\prime}\right)\right)$ and all $O \in \mathcal{K}(M, \boldsymbol{g})$. All these properties are known to hold for quasifree states of the free scalar field fulfilling the microlocal spectrum condition on global hyperbolic spacetimes, see Subsec. 3.2 for discussion.

Thus one can see that local diffeomorphism invariance really occurs at the level of local folia of states for $\mathscr{A}$. In this light, it appears natural to give a functorial description of the space of states that takes this form of local diffeomorphism invariance into account. To this end, it seems convenient to first introduce a new category, the category of the set of states.

$\mathfrak{S t s}:$ An object $\boldsymbol{S} \in \operatorname{Obj}(\mathfrak{S t s})$ is a set of states on a $C^{*}$-algebra $\mathcal{A}$. Morphisms between members $\boldsymbol{S}^{\prime}$ and $\boldsymbol{S}$ of $\operatorname{Obj}(\mathfrak{S t s})$ are positive maps $\gamma^{*}: \boldsymbol{S}^{\prime} \rightarrow \boldsymbol{S}$. In the present work, $\gamma^{*}$ arises always as the dual map of a faithful $C^{*}$-algebraic endomorphism $\gamma: \mathcal{A} \rightarrow \mathcal{A}^{\prime}$ via

$$
\gamma^{*} \omega^{\prime}(A)=\omega^{\prime}(\gamma(A)), \quad \omega^{\prime} \in \boldsymbol{S}^{\prime}, \quad A \in \mathcal{A} .
$$

The category $\mathfrak{S}$ ts is therefore "dual" to the category $\mathfrak{A} \mathfrak{d g}$. The composition rules for morphisms should thus be obvious.

Now we can define a state space for a locally covariant quantum field theory in a functorial manner.

\subsection{Definition.}

Let $\mathscr{A}$ be a locally covariant quantum field theory.

(i) A state space for $\mathscr{A}$ is a contravariant functor $\boldsymbol{S}$ between $\mathfrak{M a n}$ and $\mathfrak{S t s :}$

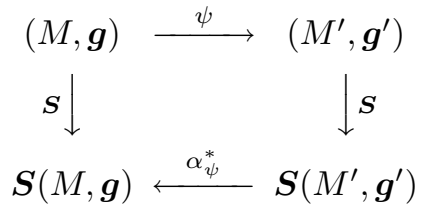

where $\boldsymbol{S}(M, \boldsymbol{g})$ is a set of states on $\mathscr{A}(M, \boldsymbol{g})$ and $\alpha_{\psi}^{*}$ is the dual map of $\alpha_{\psi}$; the contravariance property is

$$
\alpha_{\tilde{\psi} \circ \psi}^{*}=\alpha_{\psi}^{*} \circ \alpha_{\tilde{\psi}}^{*}
$$

together with the requirement that unit morphisms are mapped to unit morphisms.

(ii) We say that a state space $\boldsymbol{S}$ is locally quasi-equivalent if eqn. (6) holds for any pair of states $\omega, \tilde{\omega} \in \boldsymbol{S}(M, \boldsymbol{g})$ (with GNS-representations $\pi, \tilde{\pi}$ ) whenever $(M, \boldsymbol{g})$ and $O \in \mathcal{K}(M, \boldsymbol{g})$.

(iii) A state space $\boldsymbol{S}$ is called locally normal if there exists a locally quasiequivalent state space $\tilde{\boldsymbol{S}}$ so that for each $\omega \in \boldsymbol{S}(M, \boldsymbol{g})$ there is some $\tilde{\omega} \in \tilde{\boldsymbol{S}}(M, \boldsymbol{g})$ (with GNS-representation $\tilde{\pi}$ ) so that (可) holds for all $O \in \mathcal{K}(M, \boldsymbol{g})$.

(iv) We say that a state space $\boldsymbol{S}$ is intermediate factorial if each state $\omega \in$ $\boldsymbol{S}(M, \boldsymbol{g})$ fulfills the condition of intermediate factoriality.

We list a few direct consequences of the previous definitions.

\subsection{Theorem.}

(a) Let $\boldsymbol{S}$ be a state space which is intermediate factorial. Then for all spacetimes $(M, \boldsymbol{g}),\left(M^{\prime}, \boldsymbol{g}^{\prime}\right) \in \operatorname{Obj}(\mathfrak{M a n})$ and all pairs of states $\omega \in \boldsymbol{S}(M, \boldsymbol{g}), \omega^{\prime} \in \boldsymbol{S}\left(M^{\prime}, \boldsymbol{g}^{\prime}\right)$ with GNS-representations $\pi, \pi^{\prime}$ there holds

$$
\boldsymbol{F}\left(\pi^{\prime} \circ \alpha_{\psi} \circ \alpha_{M, O}\right)=\boldsymbol{F}\left(\pi \circ \alpha_{M, O}\right), \quad O \in \mathcal{K}(M, \boldsymbol{g}),
$$


if and only if the state space is locally quasi-equivalent.

(b) If the state space $\boldsymbol{S}$ is locally normal, then there exists a family of states $\left\{\omega_{(M, \boldsymbol{g})}:(M, \boldsymbol{g}) \in \operatorname{Obj}(\mathfrak{M a n})\right\}$ on $\mathscr{A}$ with the property that each $\omega \in \boldsymbol{S}(M, \boldsymbol{g})$ is locally normal to $\omega_{(M, \boldsymbol{g})}$.

(c) If $\tilde{\boldsymbol{S}}$ is a locally quasi-equivalent and intermediate factorial state space, then one obtains a convex, locally normal state space $\boldsymbol{S}$ by defining $\boldsymbol{S}(M, \boldsymbol{g})$ as the set of all states which are locally normal to any state on $\tilde{\boldsymbol{S}}(M, \boldsymbol{g})$.

Proof. In our proof, we will make use of the following statements:

$(\alpha)$ Let $\mathcal{A}, \mathcal{B}$ and $\mathcal{C}$ be $C^{*}$-algebras with $C^{*}$-algebraic endomorphisms

$$
\mathcal{A} \stackrel{\beta}{\longrightarrow} \mathcal{B} \stackrel{\gamma}{\longrightarrow} \mathcal{C},
$$

and let $\omega$ be a state on $\mathcal{C}$. Then there holds

$$
\boldsymbol{F}\left(\pi_{\omega} \circ \gamma \circ \beta\right) \supset \boldsymbol{F}\left(\pi_{\omega \circ \gamma} \circ \beta\right) \supset \boldsymbol{F}\left(\pi_{\omega \circ \gamma \circ \beta}\right),
$$

where $\pi_{\nu}$ denotes the GNS-representation of the state $\nu$, we will use this notation also below.

( $\beta$ ) Let $\mathcal{N}$ be a factorial von Neumann algebra on some Hilbert-space $\mathcal{H}$, and let $\mathcal{H}_{\mathcal{N}}$ be some $\mathcal{N}$-invariant closed, non-zero subspace. Then for every density matrix $\rho=\sum_{i} \lambda_{i}\left|\phi_{i}\right\rangle\left\langle\phi_{i}\right|$, where the $\phi_{i}$ are unit vectors in $\mathcal{H}$, there exists a density matrix $\rho^{\mathcal{N}}=\sum_{j} \mu_{j}\left|\chi_{j}\right\rangle\left\langle\chi_{j}\right|$, where the $\chi_{j}$ are unit vectors in $\mathcal{H}_{\mathcal{N}}$, so that

$$
\operatorname{tr}(\rho \cdot N)=\operatorname{tr}\left(\rho^{\mathcal{N}} \cdot N\right)
$$

holds for all $N \in \mathcal{N}$.

These statements will be proved in the appendix.

(a). A first immediate observation is that $\alpha_{\psi}^{*} \boldsymbol{S}\left(M^{\prime}, \boldsymbol{g}^{\prime}\right) \subset \boldsymbol{S}(M, \boldsymbol{g})$ together with the condition of local quasi-equivalence imply

$$
\boldsymbol{F}\left(\pi_{\omega^{\prime} \circ \alpha_{\psi}} \circ \alpha_{M, O}\right)=\boldsymbol{F}\left(\pi \circ \alpha_{M, O}\right), \quad O \in \mathcal{K}(M, \boldsymbol{g}) .
$$

Now fix $O \in \mathcal{K}(M, \boldsymbol{g})$. According to the assumed condition of intermediate factoriality, there are a region $O_{1} \in \mathcal{K}(M, \boldsymbol{g})$ and a factorial von Neumann algebra $\mathcal{N}$ so that

$$
\mathcal{M}_{\omega^{\prime}}(\psi(O)) \subset \mathcal{N} \subset \mathcal{M}_{\omega^{\prime}}\left(\psi\left(O_{1}\right)\right) .
$$

Consequently, if we choose an arbitrary state $\omega_{1} \in \boldsymbol{F}\left(\pi_{\omega^{\prime}} \circ \alpha_{\psi} \circ \alpha_{M, O}\right)$, then there exists, according to statement $(\beta)$ above, a density matrix $\rho^{\mathcal{N}}=\sum_{j} \mu_{j}\left|\chi_{j}\right\rangle\left\langle\chi_{j}\right|$ with $\chi_{j} \in \mathcal{H}_{\mathcal{N}}=\overline{\mathcal{N} \Omega^{\prime}}$ (where $\Omega^{\prime}$ is the GNS-vector of $\omega^{\prime}$ ) with the property

$$
\omega_{1}(A)=\operatorname{tr}\left(\rho^{\mathcal{N}} \cdot \pi_{\omega^{\prime}} \circ \alpha_{\psi} \circ \alpha_{M, O}(A)\right), \quad A \in \alpha_{M, O}\left(\mathscr{A}\left(O, \boldsymbol{g}_{O}\right)\right) .
$$

Therefore, the state is in particular given by a density matrix $\rho^{\mathcal{N}}$ in the GNSrepresentation of $\omega^{\prime} \circ \alpha_{M^{\prime}, \psi\left(O_{1}\right)}$, so that $\omega_{1}$ extends to a state

$$
\bar{\omega}_{1} \in \boldsymbol{F}\left(\pi_{\omega^{\prime} \circ \alpha_{M^{\prime}, \psi\left(O_{1}\right)}}\right) .
$$

Owing to covariance, this in turn shows that

$$
\bar{\omega}_{1} \in \boldsymbol{F}\left(\pi_{\omega^{\prime} \circ \alpha_{\psi} \circ \alpha_{M, O_{1}}}\right) \text {. }
$$

Restricting $\bar{\omega}_{1}$ again to $\omega_{1}=\bar{\omega}_{1} \circ \alpha_{M, O}$ on $\mathscr{A}\left(O, \boldsymbol{g}_{O}\right)$ yields

$$
\omega_{1} \in \boldsymbol{F}\left(\pi_{\omega^{\prime} \circ \alpha_{\psi}} \circ \alpha_{M, O}\right) \text {. }
$$

In view of statement $(\alpha)$ above and because of (10), we have thus shown that (8) holds for all $O \in \mathcal{K}(M, \boldsymbol{g})$ if $\boldsymbol{S}$ is locally quasi-equivalent. The reverse implication, saying that (8) implies that $\boldsymbol{S}$ is locally quasi-equivalent, is evident.

(b). One may choose an arbitrary family of states $\omega_{(M, \boldsymbol{g})} \in \tilde{\boldsymbol{S}}(M, \boldsymbol{g})$; since each 
such choice of states is locally quasi-equivalent to any other, by definition each state in $\boldsymbol{S}(M, \boldsymbol{g})$ will be locally normal to $\omega_{(M, \boldsymbol{g})}$.

(c). If $\boldsymbol{S}$ is a state space, then it is clearly locally normal owing to the way it is defined. So it suffices to prove that $\boldsymbol{S}$ is a state space, and convex.

To show that $\boldsymbol{S}$ is a state space, it is enough to demontrate that

$$
\alpha_{\psi}^{*}\left(\boldsymbol{S}\left(M^{\prime}, \boldsymbol{g}^{\prime}\right)\right) \subset \boldsymbol{S}(M, \boldsymbol{g}),
$$

since the contravariance property of the $\alpha_{\psi}^{*}$ 's is inherited from the covariance property of the $\alpha_{\psi}$ 's. Now if $\omega^{\prime} \in \boldsymbol{S}\left(M^{\prime}, \boldsymbol{g}^{\prime}\right)$, then this means that

$$
\omega^{\prime} \circ \alpha_{M^{\prime}, O^{\prime}} \in \boldsymbol{F}\left(\pi_{\hat{\omega}} \circ \alpha_{M^{\prime}, O^{\prime}}\right)
$$

holds for all $O^{\prime} \in \mathcal{K}\left(M^{\prime}, \boldsymbol{g}^{\prime}\right)$, where $\hat{\omega}$ is some element in $\tilde{\boldsymbol{S}}\left(M^{\prime}, \boldsymbol{g}^{\prime}\right)$. Using covariance one deduces from this relation

$$
\left(\alpha_{\psi}^{*} \omega^{\prime}\right) \circ \alpha_{M, O}=\omega^{\prime} \circ \alpha_{\psi} \circ \alpha_{M, O} \in \boldsymbol{F}\left(\pi_{\hat{\omega}} \circ \alpha_{\psi} \circ \alpha_{M, O}\right) .
$$

Then part $(a)$ of the proposition entails

$$
\left(\alpha_{\psi}^{*} \omega^{\prime}\right) \circ \alpha_{M, O} \in \boldsymbol{F}\left(\pi_{\tilde{\omega}} \circ \alpha_{M, O}\right)
$$

for all $O \in \mathcal{K}(M, \boldsymbol{g})$ with some $\tilde{\omega} \in \tilde{\boldsymbol{S}}(M, \boldsymbol{g})$, showing that $\alpha_{\psi}^{*} \omega^{\prime} \in \boldsymbol{S}(M, \boldsymbol{g})$.

Finally, we show that $\boldsymbol{S}$ is convex. Let $\omega^{\prime}=\lambda \omega_{1}+(1-\lambda) \omega_{2}$ be a convex combination of two states $\omega_{1}$ and $\omega_{2}$ in $\boldsymbol{S}(M, \boldsymbol{g})$. Then $\omega_{j} \circ \alpha_{M, O} \in \boldsymbol{F}\left(\pi_{\tilde{\omega}} \circ \alpha_{M, O}\right)$, $j=1,2$, for some state $\tilde{\boldsymbol{\omega}} \in \tilde{\boldsymbol{S}}(M, \boldsymbol{g})$, and going back to the definition of the folium, this shows in fact that $\omega^{\prime} \circ \alpha_{M, O} \in \boldsymbol{F}\left(\pi_{\tilde{\omega}} \circ \alpha_{M, O}\right)$. Thus $\omega^{\prime} \in \boldsymbol{S}(M, \boldsymbol{g})$, showing that $\boldsymbol{S}(M, \boldsymbol{g})$ is convex.

Finally, we shall demonstrate that a locally normal and intermediate factorial state space induces a generally covariant realization of the principle of local definiteness proposed by Haag, Narnhofer and Stein 20]. This principle was introduced in the context of a net of observable algebras $\{\mathcal{A}(O)\}_{O \in \mathcal{K}(M, g)}$ over a fixed, globally hyperbolic background spacetime $(M, \boldsymbol{g})$. The principle of local definiteness demands that there exists a Hilbert-space representation $\pi$ of the $C^{*}$-algebra $\mathcal{A}$ generated by $\{\mathcal{A}(O)\}_{O \in \mathcal{K}(M, g)}$ so that the set of states, $\mathcal{S}$, of the theory can be characterized as consisting of all states $\omega$ on $\mathcal{A}$ that can be extended to normal states on the local von Neumann algebras $\mathcal{M}(O)=\pi(\mathcal{A}(O))^{\prime \prime}, O \in \mathcal{K}(M, \boldsymbol{g})$. Furthermore, it was required in 20] that the local von Neumann algebras $\mathcal{M}(O)$ are factors, at least for a suitable collection of regions $O$. Here we take the point of view that one should replace this condition by the (weaker) condition of intermediate factoriality with respect to the family of local von Neumann algebras $\{\mathcal{N}(O)\}_{O \in \mathcal{K}(M, g)}$ since this avoids having to specify precise geometric conditions on the regions $O$ for which $\mathcal{M}(O)$ should be a factor.

Adopting this point of view, we may observe the following. Let $\mathscr{A}$ be a locally covariant quantum field theory with a locally normal and intermediate factorial state space $\boldsymbol{S}$, and for $(M, \boldsymbol{g}) \in \operatorname{Obj}(\mathfrak{M a n})$, let $\{\mathcal{A}(O)\}_{O \in \mathcal{K}(M, \boldsymbol{g})}$ be the net of $C^{*}$-algebras on $(M, \boldsymbol{g})$ induced by $\mathscr{A}$ according Prop. 2.2. Let $\tilde{\omega}$ be any state in $\tilde{\boldsymbol{S}}(M, \boldsymbol{g})$ where $\tilde{\boldsymbol{S}}$ is a locally quasi-equivalent state space to which $\boldsymbol{S}$ is locally normal (cf. Def. 2.3(iii)), and denote by $\tilde{\pi}$ the corresponding GNS-representation. This representation induces a representation $\pi$ of $\mathscr{A}$ via defining the representations $\pi \uparrow \mathcal{A}(O)$ as $\tilde{\pi} \circ \alpha_{M, O}^{-1}$, and hence it induces the corresponding net of von Neumann algebras $\{\mathcal{N}(O)\}_{O \in \mathcal{K}(M, \boldsymbol{g})}$. It is easy to see that each state $\omega \in \boldsymbol{S}(M, \boldsymbol{g})$ extends to a normal state on $\mathcal{M}(O)$ owing to local normality of $\boldsymbol{S}$; additionally $\{\mathcal{M}(O)\}_{O \in \mathcal{K}(M, \boldsymbol{g})}$ satisfies the condition of intermediate factoriality because $\boldsymbol{S}$ is intermediate factorial. We formulate the result of this discussion subsequently as 
3.3. Proposition. If $\boldsymbol{S}$ is locally normal and intermediate factorial, then the set of states $\boldsymbol{S}(M, \boldsymbol{g})$ for $\{\mathcal{A}(O)\}_{O \in \mathcal{K}(M, \boldsymbol{g})}$ fulfills the principle of local definiteness, for each $(M, \boldsymbol{g}) \in \operatorname{Obj}(M, \boldsymbol{g})$.

\subsection{State Space of the Klein-Gordon Field Distinguished by Microlocal Spectrum Condition.}

For the locally covariant quantum field theory of the Klein-Gordon field, we will show in the present subsection that the microlocal spectrum condition selects a state space that is locally quasi-equivalent and intermediate factorial.

We have to provide some explanations first. Let $(M, \boldsymbol{g}) \in \operatorname{Obj}(\mathfrak{M a n})$ and let $E$, $\mathfrak{W}(\mathcal{R}, \sigma)$ be defined with respect to the Klein-Gordon equatation (11) on $(M, \boldsymbol{g})$. A state $\omega$ on $\mathfrak{W}(\mathcal{R}, \sigma)$ is called quasifree if its two-point function

$$
w_{2}^{(\omega)}(f, h)=\left.\partial_{t} \partial_{\tau}\right|_{t=\tau=0} \omega(W(t E f) W(\tau E h))
$$

exists for all $f, h \in C_{0}^{\infty}(M, \mathbb{R})$, and if $\omega$ is determined by $w_{2}^{(\omega)}$ according to

$$
\omega(W(E f))=\mathrm{e}^{-w_{2}^{(\omega)}(f, f)} .
$$

A quasifree state $\omega$ is a Hadamard state if its two-point function is of Hadamard form. This property is a constraint on the short-distance behaviour of the twopoint function. Qualitatively, it means that $w_{2}^{(\omega)}$ is a distribution on $C_{0}^{\infty}(M, \mathbb{R}) \times$ $C_{0}^{\infty}(M, \mathbb{R})$ of the form

$$
w_{2}^{(\omega)}(f, h)=\lim _{\epsilon \rightarrow 0} \int\left(G_{\epsilon}(x, y)+H_{\omega}(x, y)\right) f(x) h(y) d \mu_{\boldsymbol{g}}(x) d \mu_{\boldsymbol{g}}(y)
$$

where $H_{\omega}$ is a smooth integral kernel depending on the state $\omega$, while the singular part of $w_{2}^{(\omega)}$ is given as the limit of a family of integral kernels $G_{\epsilon}$ which are determined by the metric $\boldsymbol{g}$ and the Klein-Gordon equation via the so-called Hadamard recursion relations. The leading singularity is of the type 1 /(squared geodesic distance from $x$ to $y$ ). We refer to [29 for details. The Hadamard property can be equivalently expressed in terms of a condition on the wavefront set $\mathrm{WF}\left(w_{2}^{(\omega)}\right)$ of the two-point function [32] (see also [34]): $\omega$ is a Hadamard state exactly if the pairs of covectors $(x, \eta)$ and $\left(x^{\prime}, \eta^{\prime}\right)$ which are in $\mathrm{WF}\left(w_{2}^{(\omega)}\right)$ are such that their base-points $x$ and $x^{\prime}$ lie on a lightlike geodesic, and the co-tangent vectors $\eta$ and $-\eta^{\prime}$ are co-tangent and co-parallel to that geodesic, with $\eta$ future-pointing.

This characterization of the Hadamard condition in terms of a constraint on the two-point function of a state is also referred to a "microlocal spectrum condition" because it mimicks the usual, flat space spectrum condition in the sense of microlocal analysis; its advantage is that it may be formulated for general quantum field theories, in contrast to the Hadamard condition which requires that the 2-point function satisfies a hyperbolic wave-equation [7, 41]. We refer to the indicated references for further discussion. In the context of the present subsection, we will use "Hadamard condition" and "microlocal spectrum condition" synonymously.

Now let $\mathscr{A}$ be the locally covariant quantum field theory associated with the Klei-Gordon field as in Subsec. 2.3. It is important to note that, owing to the functorial transformation properties of wavefront sets under diffeomorphisms [26, a quasifree Hadamard state $\omega^{\prime}$ on $\mathscr{A}\left(M^{\prime}, \boldsymbol{g}^{\prime}\right)$ induces a quasifree Hadamard state $\omega^{\prime} \circ \alpha_{\psi}$ on $\mathscr{A}(M, g)$ whenever $\psi \in \operatorname{hom}_{\mathfrak{M a n}}\left((M, \boldsymbol{g}),\left(M^{\prime}, \boldsymbol{g}^{\prime}\right)\right)$. Furthermore, was shown in [18] that there exists a large set of quasifree Hadamard states for the Klein-Gordon field on every globally hyperbolic spacetime $(M, \boldsymbol{g})$. Moreover, the results in [39] show that the GNS-representations of quasifree Hadamard states are locally quasi-equivalent, and in 40 it was proved that the condition of intermediate factoriality is fulfilled for quasifree Hadamard states. We may thus summarize these results in the subsequent 
3.4. Theorem. For each $(M, \boldsymbol{g}) \in \operatorname{Obj}(\mathfrak{M a n})$, define $\boldsymbol{S}(M, \boldsymbol{g})$ as the set of all states on $\mathscr{A}(M, \boldsymbol{g})$ whose GNS-representations are locally quasiequivalent to the GNS-representation of any quasifree Hadamard state on $\mathscr{A}(M, \boldsymbol{g})$. This assignment results in a state space which is locally quasi-equivalent and intermediate factorial, and $\boldsymbol{S}(M, \boldsymbol{g})$ contains in particular all quasifree Hadamard states on $\mathscr{A}(M, \boldsymbol{g})$.

\section{Dynamics}

\subsection{Relative Cauchy-Evolution.}

For theories obeying the time-slice axiom one can define relative Cauchy-evolutions, as follows. Let $\left(M_{1}, \boldsymbol{g}_{1}\right)$ and $\left(M_{2}, \boldsymbol{g}_{2}\right)$ be in $\operatorname{Obj}(\mathfrak{M a n})$. We suppose that there are globally hyperbolic sub-regions $N_{j}^{ \pm}$of $M_{j}, j=1,2$ containing Cauchy-surfaces of the respective spacetimes. Moreover, we assume that there are isometric (and orientation/time-orientation-preserving) diffeomorphisms $\iota^{ \pm}: N_{1}^{ \pm} \rightarrow N_{2}^{ \pm}$when the regions are endowed with the appropriate restrictions of the metrics $\boldsymbol{g}_{1}$ and $\boldsymbol{g}_{2}$, respectively. Henceforth, we shall suppress the diffeomorphisms $\iota^{ \pm}$in our notation and identify $N_{1}^{ \pm}$and $N_{2}^{ \pm}$as being equal. The isometric embeddings of $N_{j}^{ \pm}$into $M_{j}$ will be denoted by $\psi_{j}^{ \pm}$. They are depicted in the following diagram:

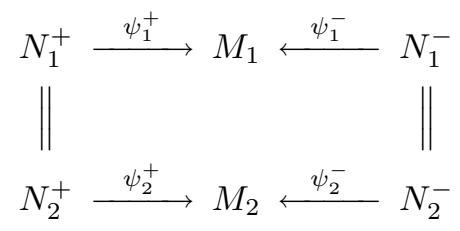

By the functorial properties of a locally covariant quantum field theory $\mathscr{A}$, the previous diagram gives rise to the next:

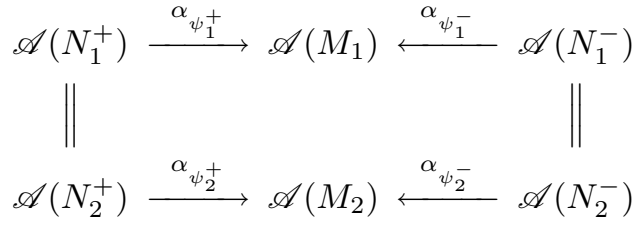

where we have, for the sake of simplicity, suppressed the appearence of the spacetime metrics in our notation. If the theory $\mathscr{A}$ obeys the time-slice axiom, then all the morphisms in this diagram are onto and invertible, and hence one obtains from it an automorphism $\beta \in \operatorname{hom}_{\mathfrak{A} \mathfrak{g} \mathfrak{g}}\left(\mathscr{A}\left(M_{1}\right), \mathscr{A}\left(M_{1}\right)\right)$ by setting

$$
\beta=\alpha_{\psi_{1}^{-}} \circ \alpha_{\psi_{2}^{-}}^{-1} \circ \alpha_{\psi_{2}^{+}} \circ \alpha_{\psi_{1}^{+}}^{-1} \text {. }
$$

Under certain circumstances (which may be expected to be generically fulfilled) it is possible to form the functional derivative of the relative Cauchy-evolution with respect to the metrics of the spacetimes involved in its construction. This functional derivative then has the meaning of an energy-momentum tensor. In fact, we will show below for the example of the Klein-Gordon field that the functional derivative of the relative Cauchy-evolution agrees with the action of the quantized energymomentum tensor in representations of quasifree Hadamard states.

In order to give these ideas a more precise shape, we introduce the following

\section{Geometric assumptions.}

- We consider a globally hyperbolic spacetime $(M, \stackrel{\circ}{\boldsymbol{g}})$ where it is assumed that $M$ can be covered by a single coordinate patch.

- We pick a Cauchy-surface $C$ in $(M, \stackrel{\circ}{\boldsymbol{g}})$, and two open subregions $N_{ \pm}$of $M$ with the properties:

$$
-\overline{N_{ \pm}} \subset \operatorname{int} J^{ \pm}(C)
$$


- $\left(N_{ \pm},{\stackrel{\circ}{g}}_{N_{ \pm}}\right)$are contained in $\operatorname{Obj}(\mathfrak{M a n})$,

- $N_{ \pm}$contain Cauchy-surfaces for $(M, \stackrel{\circ}{g})$.

- Let $G$ be a set of Lorentzian metrics on $M$ with the following properties:

- Each $\boldsymbol{g} \in G$ deviates from $\stackrel{\circ}{g}$ only on a compact subset of the region

$$
M_{(+,-)}=M \backslash \operatorname{cl}\left[J^{-}\left(N_{-}\right) \cup J^{+}\left(N_{+}\right)\right]
$$

- each $(M, \boldsymbol{g}), \boldsymbol{g} \in G$, is a member of $\operatorname{Obj}(\mathfrak{M a n})$,

- $C$ is a Cauchy-surface for $(M, \boldsymbol{g}), \boldsymbol{g} \in G$,

- If $\boldsymbol{g} \in G$, then the family $\left\{\boldsymbol{g}_{s}\right\}_{s \in[0,1]}$ of metrics given by

$$
\boldsymbol{g}_{s}=\stackrel{\circ}{\boldsymbol{g}}+s(\boldsymbol{g}-\stackrel{\circ}{\boldsymbol{g}}), \quad 1 \geq s \geq 0,
$$

is also contained in $G$.

- If $\phi$ is a diffeomorphism of $M$ which acts trivially outside of $M_{(+,-)}$, then $\phi_{*} \boldsymbol{g}$ is contained in $G$.

It is clear from these assumptions that one may view the metrics $\boldsymbol{g}$ in $G$ as "perturbations" around the metric $\stackrel{\circ}{\boldsymbol{g}}$ on $M_{(+,-)}$. Moreover, $\left(N_{ \pm},{\stackrel{\circ}{N_{N}}}\right)$ are also globally hyperbolic submanifolds of $(M, \boldsymbol{g})$ for each $\boldsymbol{g} \in G$. Hence there are isometric embeddings $\psi_{\boldsymbol{g}}^{ \pm} \in \operatorname{hom}_{\mathfrak{M a n}}\left(\left(N_{ \pm},{\stackrel{\circ}{\boldsymbol{g}_{N}}}_{N_{ \pm}}\right),(M, \boldsymbol{g})\right)$ for all $\boldsymbol{g} \in G$ as well as isometric embeddings $\psi_{\circ}^{ \pm} \in \operatorname{hom}_{\mathfrak{M a n}}\left(\left(N_{ \pm},{\stackrel{\circ}{\boldsymbol{g}}}_{N_{ \pm}}\right),(M, \stackrel{\circ}{\boldsymbol{g}})\right)$. To these embeddings one can associate the relative Cauchy-evolution $\beta_{\boldsymbol{g}} \in \operatorname{hom}_{\mathfrak{A} \mathfrak{g}}(\mathscr{A}(M, \stackrel{\circ}{\boldsymbol{g}}), \mathscr{A}(M, \stackrel{\circ}{\boldsymbol{g}}))$ given by

$$
\beta_{\boldsymbol{g}}=\alpha_{\psi_{\mathrm{o}}^{-}} \circ \alpha_{\psi_{\boldsymbol{g}}^{-}}^{-1} \circ \alpha_{\psi_{\boldsymbol{g}}^{+}} \circ \alpha_{\psi_{\circ}^{+}}^{-1}
$$

Remarks. (A) One may view $\beta_{\boldsymbol{g}}$ as a "scattering morphism" describing the change that the propagation of a quantum field undergoes passing through the region with the "metric perturbation" $\boldsymbol{g}-\stackrel{\circ}{g}$ compared to the background metric $\stackrel{\circ}{\boldsymbol{g}}$.

(B) There is some relation between the relative Cauchy-evolution and the evolution of Cauchy-data from one Cauchy-surface to another which e.g. in the case of the scalar Klein-Gordon field is also known to lead to $C^{*}$-algebraic endomorphisms [28, 37. We refer to the references for more discussion.

(C) Hollands and Wald 24 consider for the case of the free Klein-Gordon field related operators $\tau_{\boldsymbol{g}}^{\text {adv }}$ and $\tau_{\boldsymbol{g}}^{\text {ret }}$, which would correspond to the operators $\alpha_{\psi_{\circ}^{+}} \circ \alpha_{\psi_{\boldsymbol{g}}^{+}}^{-1}$ and $\alpha_{\psi_{0}^{-}} \circ \alpha_{\psi_{g}^{-}}^{-1}$.

As the theory $\mathscr{A}$ is locally covariant, it follows that the relative Cauchy-evolution is insensitive to changing $\boldsymbol{g}$ into $\phi_{*} \boldsymbol{g}$ when $\phi$ is a diffeomorphism of $M$ that acts trivially outside of the intermediate region $M_{(+,-)}$. More precisely, one obtains:

4.1. Proposition. Let $\phi$ be a diffeomorphism of $M$ that acts trivially outside of $M_{(+,-)}$(i.e. $\phi(x)=x$ for all $x$ in the complement of $\left.M_{(+,-)}\right)$. Then

$$
\beta_{\boldsymbol{g}}=\beta_{\phi_{*} \boldsymbol{g}}, \quad \boldsymbol{g} \in G .
$$

Proof. It holds that $\phi$ is a morphism in $\operatorname{hom}_{\mathfrak{M a n}}\left((M, \boldsymbol{g}),\left(M, \phi_{*} \boldsymbol{g}\right)\right)$, and hence

$$
\phi \circ \psi_{\boldsymbol{g}}^{ \pm}=\psi_{\phi_{*} \boldsymbol{g}}^{ \pm}
$$


owing to the definition of $\psi_{\boldsymbol{g}}^{ \pm}$since $\phi$ acts trivially on $N_{ \pm}$. On the other hand, it holds that

$$
\begin{aligned}
\beta_{\boldsymbol{g}} & =\alpha_{\psi_{\circ}^{-}} \circ \alpha_{\psi_{\boldsymbol{g}}^{-}}^{-1} \circ \alpha_{\psi_{\boldsymbol{g}}^{+}} \circ \alpha_{\psi_{\circ}^{+}}^{-1} \\
& =\alpha_{\psi_{\circ}^{-}} \circ \alpha_{\psi_{\boldsymbol{g}}^{-}}^{-1} \circ \alpha_{\phi}^{-1} \circ \alpha_{\phi} \circ \alpha_{\psi_{\boldsymbol{g}}^{+}} \circ \alpha_{\psi_{\circ}^{+}}^{-1} \\
& =\alpha_{\psi_{\circ}^{-}} \circ \alpha_{\phi \circ \psi_{\boldsymbol{g}}^{-}}^{-1} \circ \alpha_{\phi \circ \psi_{\boldsymbol{g}}^{+}} \circ \alpha_{\psi_{0}^{+}}^{-1} \\
& =\alpha_{\psi_{\circ}^{-}} \circ \alpha_{\psi_{\phi_{*} g}^{-}}^{-1} \circ \alpha_{\psi_{\phi_{*}}^{+}} \circ \alpha_{\psi_{\circ}^{+}}^{-1} \\
& =\beta_{\phi_{*} \boldsymbol{g}} .
\end{aligned}
$$

We will now make assumptions that allow us to define the functional derivative of $\beta_{\boldsymbol{g}}$ with respect to $\boldsymbol{g} \in G$. To this end, we assume that $\pi$ is a Hilbert-space representation of $\mathscr{A}(M, \stackrel{\circ}{g})$, and that there is a dense subspace $\mathcal{V}$ of the representationHilbert-space $\mathcal{H}$ and a dense $*$-sub-algebra $\mathcal{B}$ of $\mathscr{A}(M, \stackrel{\circ}{\boldsymbol{g}})$ so that, for all smooth curves $[0,1] \ni s \mapsto \boldsymbol{g}_{s} \in G$ with $\boldsymbol{g}_{(s=0)}=\stackrel{\circ}{\boldsymbol{g}}$, there holds

$$
\left.\frac{d}{d s}\left\langle\theta, \pi\left(\beta_{\boldsymbol{g}_{s}}(B)\right) \theta\right\rangle\right|_{s=0}=\int_{M} b^{\mu \nu}(x) \delta \boldsymbol{g}_{\mu \nu}(x)(-\stackrel{\circ}{g}(x))^{1 / 2} d x
$$

for all $\theta \in \mathcal{V}, B \in \mathcal{B}$ with a suitable smooth section $x \mapsto b^{\mu \nu}(x)$ in $T M \otimes T M$ (depending on $\theta$ and $B$ ); we have written $\delta \boldsymbol{g}=d \boldsymbol{g}_{s} /\left.d s\right|_{s=0}$, and $\stackrel{\circ}{g}$ is the determinant of $\stackrel{\circ}{g}$ in the coordinates used for $M$. Then we write

$$
\left\langle\theta, \frac{\delta}{\delta \boldsymbol{g}_{\mu \nu}(x)} \pi\left(\beta_{\boldsymbol{g}} B\right) \theta\right\rangle=b^{\mu \nu}(x),
$$

and thus the functional derivative of the relative Cauchy-evolution $\beta_{\boldsymbol{g}}$ with respect to the metric $\boldsymbol{g}$,

$$
\frac{\delta}{\delta \boldsymbol{g}_{\mu \nu}(x)} \pi\left(\beta_{\boldsymbol{g}} B\right)
$$

is defined in the representation $\pi$ for all $B \in \mathcal{B}$ in the sense of quadratic forms on $\mathcal{V}$. (As announced before, these assumptions are realized for the free scalar Klein-Gordon field in representations of quasifree Hadamard states, see Sec. 4.2 below). The functional derivative of $\beta_{\boldsymbol{g}}$ with respect to $\boldsymbol{g}$ describes the reaction of the quantum system to an infinitesimal local change of the spacetime metric. As known in classical field theory, this is described by the energy-momentum tensor, and we will find this corroborated in the quantum field case by Thm 4.3 below. It is mentioned in 24 that the functional derivative of $\tau_{\boldsymbol{g}}^{\text {adv/ret }}$ with respect to $\boldsymbol{g}$ describes the advanced/retarded response of the quantum system upon infinitesimal metric changes.

When the indicated assumptions are fulfilled, then we find that the relative Cauchy-evolution is divergence-free.

4.2. Theorem. For all $B \in \mathcal{B}$, one has

$$
\nabla_{\mu} \frac{\delta}{\delta \boldsymbol{g}_{\mu \nu}(x)} \pi\left(\beta_{\boldsymbol{g}}(B)\right)=0, \quad x \in M,
$$

in the sense of quadratic forms on $\mathcal{V}$ where $\nabla$ is the covariant derivative with respect to $\stackrel{\circ}{\mathrm{g}}$.

Proof. Let $X$ be a smooth vector field on $M$ which vanishes outside of $M_{(+,-)}$, and let $\phi_{s}, s \in \mathbb{R}$, be the one-parametric group of diffeomorphisms that is generated by 
$X$. By Prop. 4.1, we have $\beta_{\mathrm{g}}-\beta_{\phi_{s *} \stackrel{\circ}{ }}=0$ for all $s$, and hence one obtains that

$$
\frac{d}{d s} \beta_{\phi_{s *} \stackrel{\circ}{g}}=0 .
$$

On the other hand, using the notation $b^{\mu \nu}(x)=\left\langle\theta, \delta \pi\left(\beta_{\boldsymbol{g}}(B)\right) / \delta \boldsymbol{g}_{\mu \nu}(x) \theta\right\rangle$ and recalling the definition of $\delta \beta_{\boldsymbol{g}} / \delta \boldsymbol{g}_{\mu \nu}(x)$, we have

$$
0=\left.\frac{d}{d s}\left\langle\theta, \pi\left(\beta_{\phi_{s *} \stackrel{\circ}{\boldsymbol{g}}}(B)\right) \theta\right\rangle\right|_{s=0}=\left.\int_{M} b^{\mu \nu}(x) \frac{d}{d s}\right|_{s=0} \phi_{s *} \stackrel{\circ}{\boldsymbol{g}}_{\mu \nu}(x)(-\stackrel{\circ}{g})^{1 / 2}(x) d x
$$

for all $B \in \mathcal{B}, \theta \in \mathcal{V}$. Now one can conclude that $\nabla_{\mu} b^{\mu \nu}=0$ as in the case of classical field theory (cf. [23], Sec. 3.3): It holds that $\left.\frac{d}{d s}\right|_{s=0} \phi_{s *} \stackrel{\circ}{\boldsymbol{g}}_{\mu \nu}=£_{X} \stackrel{\circ}{\boldsymbol{g}}_{\mu \nu}=$ $\nabla_{\mu} X_{\nu}+\nabla_{\nu} X_{\mu}$, where $£_{X}$ denotes the Lie-derivative, and hence

$$
\begin{aligned}
0 & =\int_{M} b^{\mu \nu}(x) £_{X}{\stackrel{\circ}{\boldsymbol{g}_{\mu \nu}}}(x)(-\stackrel{\circ}{g})^{1 / 2}(x) d x \\
& =2 \int_{M}\left(\nabla_{\mu}\left(b^{\mu \nu} X_{\nu}\right)(x)-\left(\nabla_{\mu} b^{\mu \nu}(x)\right) X_{\nu}(x)\right)(-\stackrel{\circ}{g})^{1 / 2}(x) d x .
\end{aligned}
$$

The first term in the last expression is a divergence and can be converted to a surface integral which hence vanishes since $X$ has compact support. As $X$ was an arbitrary vectorfield supported inside $M_{(+,-)}$, one thus concludes that $\nabla_{\mu} b^{\mu \nu}(x)=0$ for $x \in M_{(+,-)}$; on the other hand, $b^{\mu \nu}(x)=0$ for all $x$ outside of $M_{(+,-)}$according to the definition of the functional derivative of the Cauchy-evolution. Thus $\nabla_{\mu} b^{\mu \nu}=0$ on $M$, and this completes the proof.

\subsection{Relative Cauchy-Evolution for the Klein-Gordon Field.}

In the present sub-section we will investigate the relation between the functional derivative of the relative Cauchy-evolution for the quantum Klein-Gordon field with respect to the spacetime metric, and the quantum field's energy-momentum tensor. This will be the presented in Theorem 4.3 below. Before stating this result, we will discuss the form of the relative Cauchy-evolution for the generally covariant Klein-Gordon field in some detail.

Let $(M, \boldsymbol{g})$ be an object in $\operatorname{Obj}(\mathfrak{M a n})$ and let $\left(N, \boldsymbol{g}_{N}\right)$ be globally hyperbolic sub-spacetime of $(M, \boldsymbol{g})$, so that the identical injection $\iota_{N}: N \rightarrow M, \iota_{N}(x)=x$ is a morphism in hom $\operatorname{man}_{\mathfrak{m a n}}\left(\left(N, \boldsymbol{g}_{N}\right),(M, \boldsymbol{g})\right)$, where $\boldsymbol{g}_{N}$ is $\boldsymbol{g}$ restricted to $N$. Furthermore, let $(\mathcal{R}, \sigma)$ denote the symplectic space of solutions of the Klein-Gordon equation (11) on $(M, \boldsymbol{g})$, and $\left(\mathcal{R}_{N}, \sigma_{N}\right)$ the corresponding symplectic space of solutions on $\left(N, \boldsymbol{g}_{N}\right)$. $E$ and $E_{N}$ will denote the associated propagators, respectively. We have seen above that $\iota_{N}$ induces a $C^{*}$-endomorphism $\alpha_{\iota_{N}}: \mathfrak{W}\left(\mathcal{R}_{N}, \sigma_{N}\right) \rightarrow \mathfrak{W}(\mathcal{R}, \sigma)$ by

$$
\alpha_{\iota_{N}}\left(W_{N}(\varphi)\right)=W\left(T_{N} \varphi\right), \quad \varphi \in \mathcal{R}_{N}
$$

where we have denoted by $W_{N}\left(\right.$. ) the Weyl-generators of $\mathfrak{W}\left(\mathcal{R}_{N}, \sigma_{N}\right)$ and by $W($. $)$ those of $(\mathcal{R}, \sigma)$. The map $T_{N}$ assigns to each element $E_{N} f, f \in C_{0}^{\infty}(N, \mathbb{R})$, of $\mathcal{R}_{N}$ the element $E f \in \mathcal{R}$.

Let us now consider the case where $N$ contains a Cauchy-surface for $(M, \boldsymbol{g})$. In this case, Dimock [10] has shown that the map $T_{N}$ is surjective, i.e. $T_{N} \mathcal{R}_{N}=\mathcal{R}$. $T_{N}$ is also injective (since it is symplectic), and we want to derive the form of the inverse map $T_{N}^{-1}$. To this end, let $\varphi \in \mathcal{R}$, and let $\Sigma$ be a Cauchy-surface for $(M, \boldsymbol{g})$ contained in $N$. There exists a pair of two other Cauchy-surfaces $\Sigma^{\text {adv }}$ and $\Sigma^{\text {ret }}$ for $(M, \boldsymbol{g})$ in $N$ where $\Sigma^{\text {adv }}$ lies in the timelike future and $\Sigma^{\text {ret }}$ in the timelike past of $\Sigma$, hence $U=\operatorname{int} J^{-}\left(\Sigma^{\text {adv }}\right) \cap J^{+}\left(\Sigma^{\text {ret }}\right)$ is an open neighbourhood of $\Sigma$ whose closure in contained in $N$. Now we choose a partition of unity $\left\{\chi^{\text {adv }}, \chi^{\text {ret }}\right\}$ of $M$ 
so that $\chi^{\text {adv }}=0$ on $J^{-}\left(\Sigma^{\text {ret }}\right)$ and $\chi^{\text {ret }}=0$ on $J^{+}\left(\Sigma^{\text {adv }}\right)$. Then the properties $\chi^{\mathrm{adv}}+\chi^{\mathrm{ret}}=1$ and $\left(\nabla^{a} \nabla_{a}+\xi R+m^{2}\right) \varphi=0$ imply

$$
\left(\nabla^{a} \nabla_{a}+\xi R+m^{2}\right)\left(\chi^{\text {adv }} \varphi\right)=-\left(\nabla^{a} \nabla_{a}+\xi R+m^{2}\right)\left(\chi^{\text {ret }} \varphi\right) .
$$

Since the left-hand side vanishes on $J^{-}\left(\Sigma^{\text {ret }}\right)$ and the right-hand side vanishes on $J^{+}\left(\Sigma^{\text {adv }}\right)$ while $\varphi=E f$ has support in $J(\operatorname{supp} f)$ for some compactly supported $f$, one deduces that both the left- and right-hand side expressions of (13) are compactly supported in $\bar{U} \subset N$. Using the properties of the propagator $E$, one can moreover show (cf. [10])

$$
E\left(\nabla^{a} \nabla_{a}+\xi R+m^{2}\right)\left(\chi^{\mathrm{adv} / \mathrm{ret}} \varphi\right)= \pm \varphi, \quad \varphi \in \mathcal{R} .
$$

Since $E\left(\nabla^{\mu} \nabla_{\mu}+m^{2}+\xi R\right)\left(\chi^{\text {adv } / \text { ret }} \varphi\right)$ is contained in $E\left(C_{0}^{\infty}(N, \mathbb{R})\right)$ and $E f \mapsto E_{N} f$, $f \in C_{0}^{\infty}(N, \mathbb{R})$, is a symplectic map from $(\mathcal{R}, \sigma)$ onto $\left(\mathcal{R}_{N}, \sigma_{N}\right)$ owing to the uniqueness of advanced and retarded fundamental solutions of the Klein-Gordon equation in globally hyperbolic spacetimes, we can see that $T_{N}^{-1}:(\mathcal{R}, \sigma) \rightarrow\left(\mathcal{R}_{N}, \sigma_{N}\right)$ is given by

$$
T_{N}^{-1}(\varphi)= \pm E_{N}\left(\nabla^{a} \nabla_{a}+\xi R+m^{2}\right)\left(\chi^{\mathrm{adv} / \mathrm{ret}} \varphi\right) .
$$

Now we wish to study the relative Cauchy-evolution for the scalar Klein-Gordon field. We assume that we are in the situation described in the previous subsection: We are given a globally spacetime $(M, \stackrel{\circ}{g})$, with subregions $N_{ \pm}$and $M_{(+,-)}$on the latter of which metrics $\boldsymbol{g}$ in a set $G$ deviate from $\stackrel{\circ}{g}$, where these data are subject to the geometric assumptions listed above.

For the generally covariant theory of the Klein-Gordon field, we see from our discussion above that $\beta_{\boldsymbol{g}}$ acts on the generators $\stackrel{\circ}{W}($.$) of the CCR-algebra of the$ Klein-Gordon field on $(M, \stackrel{\circ}{g})$ like

$$
\beta_{\boldsymbol{g}}(\stackrel{\circ}{W}(\varphi))=\stackrel{\circ}{W}\left(F_{\boldsymbol{g}} \varphi\right) ;
$$

here, $F_{\boldsymbol{g}}: \stackrel{\circ}{\mathcal{R}} \rightarrow \stackrel{\circ}{\mathcal{R}}$ is the symplectic map

$$
F_{\boldsymbol{g}}=T_{N_{-}, \circ} \circ T_{N_{-}, \boldsymbol{g}}^{-1} \circ T_{N_{+}, \boldsymbol{g}} \circ T_{N_{+}, \circ}^{-1}
$$

with

$$
\begin{gathered}
T_{N_{ \pm}, \boldsymbol{g}}: E_{N_{ \pm}, \boldsymbol{g}} f \mapsto E_{\boldsymbol{g}} \iota_{N_{ \pm} *} f, \quad f \in C_{0}^{\infty}\left(N_{ \pm}, \mathbb{R}\right), \\
T_{N_{ \pm}, \circ}: \stackrel{\circ}{E}_{N_{ \pm}} f \mapsto \stackrel{\circ}{E} \iota_{N_{ \pm} *} f, \quad f \in C_{0}^{\infty}\left(N_{ \pm}, \mathbb{R}\right), \\
T_{N_{ \pm}, \boldsymbol{g}}^{-1}: \phi \mapsto-E_{N_{ \pm}, \boldsymbol{g}} K_{\boldsymbol{g}}\left(\chi_{ \pm}^{\mathrm{ret}} \phi\right), \quad \phi \in \mathcal{R}_{\boldsymbol{g}}, \\
T_{N_{ \pm}, \circ}^{-1}: \varphi \mapsto-\stackrel{\circ}{E}_{N_{ \pm}} \stackrel{\circ}{K}\left(\chi_{ \pm}^{\mathrm{ret}} \varphi\right), \quad \varphi \in \stackrel{\circ}{\mathcal{R}},
\end{gathered}
$$

where $\stackrel{\circ}{E}, \stackrel{\circ}{\mathcal{R}}, \stackrel{\circ}{\sigma}, \stackrel{\circ}{E}_{N_{ \pm}}, \stackrel{\circ}{\mathcal{R}}_{N_{ \pm}}, \stackrel{\circ}{\sigma}_{N_{ \pm}}, E_{\boldsymbol{g}}, \mathcal{R}_{\boldsymbol{g}}, \sigma_{\boldsymbol{g}}$ and $E_{N_{ \pm}, \boldsymbol{g}}, \mathcal{R}_{N_{ \pm}, \boldsymbol{g}}, \sigma_{N_{ \pm}, \boldsymbol{g}}$ denote the propagators, range-spaces and symplectic forms corresponding to the Klein-Gordon equation on the spacetimes $(M, \stackrel{\circ}{\boldsymbol{g}}),\left(N_{ \pm}, \stackrel{\circ}{\boldsymbol{g}}_{N_{ \pm}}\right),(M, \boldsymbol{g})$ and $(M, \stackrel{\circ}{\boldsymbol{g}})$, respectively. The functions $\chi_{ \pm}^{\text {adv } / \text { ret }}$ are defined relative to suitable pairs of Cauchy-surfaces $\Sigma_{ \pm}^{\text {adv }} /$ ret lying in $N_{ \pm}$. By $K_{\boldsymbol{g}}$ and $\stackrel{\circ}{K}$ we denote the Klein-Gordon operator

$$
\nabla^{a} \nabla_{a}+\xi R+m^{2}
$$

on the spacetimes $(M, \boldsymbol{g})$ and $(M, \stackrel{\circ}{\boldsymbol{g}})$, respectively. Note that (up to identification) $E_{N_{ \pm}, \boldsymbol{g}}=\stackrel{\circ}{E}_{N_{ \pm}}$for all $\boldsymbol{g} \in G$ according to our geometric assumptions, and thus also 
$\mathcal{R}_{N_{ \pm}, \boldsymbol{g}}=\stackrel{\circ}{\mathcal{R}}_{N_{ \pm}}, \sigma_{N_{ \pm}, \boldsymbol{g}}=\stackrel{\circ}{\sigma}_{N_{ \pm}}$. This entails

$$
F_{\boldsymbol{g}} \varphi=\stackrel{\circ}{E} K_{\boldsymbol{g}} \chi_{-}^{\mathrm{ret}} E_{\boldsymbol{g}} \stackrel{\circ}{K} \chi_{+}^{\mathrm{ret}} \varphi, \quad \varphi \in \stackrel{\circ}{\mathcal{R}},
$$

where we have dropped the embedding identifications $\iota_{N_{ \pm} *}$ from our notation. This relation will be the key ingredient in the proof of the next theorem. Prior to stating it, some further preparation is required.

Let us select some arbitrary quasifree Hadamard state $\omega$ on $\mathscr{A}(M, \stackrel{\circ}{\boldsymbol{g}})=\mathfrak{W}(\stackrel{\circ}{\mathcal{R}}, \stackrel{\circ}{\sigma})$, the Weyl-algebra of the Klein-Gordon field on $(M, \stackrel{\circ}{g})$. Then we will write

$$
W_{\omega}(\varphi)=\pi_{\omega}(\stackrel{\circ}{W}(\varphi)), \quad \varphi \in \stackrel{\circ}{\mathcal{R}}
$$

for the Weyl-operators in the GNS-representation $\pi_{\omega}$ of $\omega$; then we have

$$
W_{\omega}(\varphi)=\mathrm{e}^{i \check{\Phi}_{\omega}(\varphi)}
$$

with suitable selfadjoint operators $\check{\Phi}_{\omega}(\varphi)$ in the GNS-Hilbert-space $\mathcal{H}_{\omega}$, depending linearly on $\varphi$, and

$$
w_{2}^{(\omega)}(f, h)=\left\langle\Omega_{\omega}, \check{\Phi}_{\omega}(\stackrel{\circ}{E} f) \check{\Phi}_{\omega}(\stackrel{\circ}{E} h) \Omega_{\omega}\right\rangle, \quad f, h \in C_{0}^{\infty}(M, \mathbb{R}),
$$

with the GNS-vector $\Omega_{\omega}$. Let $\mathcal{V}_{\omega}$ the set of all vectors $\theta$ in $\mathcal{H}_{\omega}$ which are of the form $\theta=B \Omega_{\omega}$ where $B$ is an arbitrary polynomial in the variables $W_{\omega}(\varphi), \check{\Phi}_{\omega}\left(\varphi^{\prime}\right)$ as $\varphi$ and $\varphi^{\prime}$ vary over $\stackrel{\circ}{\mathcal{R}}$. One can show that each $\theta \in \mathcal{V}_{\omega}$ is in the domain of all operators $\Phi_{\omega}(\varphi)$ and that the wavefront sets $\operatorname{WF}\left(w_{2}^{[\theta]}\right)$ of the two-point functions induced by $\theta \in \mathcal{V}_{\omega}$,

$$
w_{2}^{[\theta]}(f, h)=\left\langle\theta, \check{\Phi}_{\omega}(\stackrel{\circ}{E} f) \check{\Phi}_{\omega}(\stackrel{\circ}{E} h) \theta\right\rangle, \quad f, h \in C_{0}^{\infty}(M, \mathbb{R}),
$$

are of the same shape as those of the two-point functions of Hadamard states 15 . Furthermore, denoting by

$$
\Phi_{\omega}(f)=\check{\Phi}_{\omega}(E f), \quad f \in C_{0}^{\infty}(M, \mathbb{R}),
$$

the quantum field induced by $\check{\Phi}_{\omega}$, one can show that there is for each pair of vectors $\theta, \theta^{\prime} \in \mathcal{V}_{\omega}$ a smooth function $x \mapsto\left\langle\theta, \Phi_{\omega}(x) \theta^{\prime}\right\rangle$ on $M$ so that

$$
\left\langle\theta, \Phi_{\omega}(f) \theta^{\prime}\right\rangle=\int_{M}\left\langle\theta, \Phi_{\omega}(x) \theta^{\prime}\right\rangle f(x)(-\stackrel{\circ}{g})^{1 / 2}(x) d x
$$

where we recall that $\stackrel{\circ}{g}(x)$ is the determinant of $\stackrel{\circ}{g}$ in the coordinates used for $M$. These assertions rest on the fact that (1) $\Omega_{\omega}$ is an analytic vector for all $\check{\Phi}_{\omega}(\varphi)$, (2) $\left[\check{\Phi}_{\omega}(\varphi), W_{\omega}(\tilde{\varphi})\right]=-\sigma(\varphi, \tilde{\varphi}) W_{\omega}(\tilde{\varphi})$, and iterated use of this relation, (3) the distribution $f \mapsto w_{2}^{(\omega)}(f, h)$ is induced by a smooth function, and $w_{2}^{[\theta]}(f, h)$ can be reduced to a sum of products of such $w_{2}^{(\omega)}\left(f, h_{j}\right)$ (with suitable coefficients) since $\omega$ is quasifree.

After these preparations, we obtain:

4.3. Theorem. Under the geometric assumptions listed above, there holds

$$
\frac{\delta}{\delta \boldsymbol{g}_{\mu \nu}(x)}\left(\beta_{\boldsymbol{g}} \stackrel{\circ}{W}\right)_{\omega}(\varphi)=-\frac{i}{2}\left[T^{\mu \nu}(x), W_{\omega}(\varphi)\right], \quad \varphi \in \stackrel{\circ}{\mathcal{R}}, x \in M_{(+,-)},
$$

in the sense of quadratic forms on $\mathcal{V}_{\omega}$, where $T_{\mu \nu}$ is the generally covariant renormalized energy-momentum tensor of the quantized Klein-Gordon field on $(M, \stackrel{\circ}{\boldsymbol{g}})$ in the GNS-representation of $\omega$, and $\omega$ is an arbitrary quasifree Hadamard state. 
Remarks. (A) Note that the classical expression for $T_{\mu \nu}$ is $T_{\mu \nu}=\left.\frac{2}{\sqrt{-g}} \frac{\delta}{\delta \boldsymbol{g}^{\mu \nu}} S_{\mathrm{KG}}\right|_{\boldsymbol{g}=\stackrel{\circ}{\boldsymbol{g}}}$ where $S_{\mathrm{KG}}$ is the action integral of the Lagrangian density

$$
\mathcal{L}_{\mathrm{KG}}=\frac{1}{2} \sqrt{-g}\left(\boldsymbol{g}^{\mu \nu} \nabla_{\mu} \varphi \nabla_{\nu} \varphi-\left(m^{2}+\xi R\right) \varphi^{2}\right) .
$$

Here we use the convention that $T_{\mu \nu}$ is defined in this way, and that $T^{\mu \nu}=$ $\stackrel{\circ}{\boldsymbol{g}}^{\mu \alpha} \stackrel{\circ}{\boldsymbol{g}^{\nu \beta}} T_{\alpha \beta}$ and not $T^{\mu \nu}=\left.\frac{2}{\sqrt{-g}} \frac{\delta}{\delta \boldsymbol{g}_{\mu \nu}} S_{\mathrm{KG}}\right|_{\boldsymbol{g}=\stackrel{\circ}{\boldsymbol{g}}}$. The latter expression differs from the former, which we use, by a sign.

(B) Instead of the generally covariant renormalized energy-momentum tensor one may also use the energy-momentum tensor renormalized with respect to $\omega$ as reference state, since the two definitions differ by a term which is a multiple of the unit operator and hence is cancelled by the commutator on the right hand side of (15). In fact, one may even use (after point-split regularization) the "unrenormalized, formal expression" (cf. [14]) for the quantum energy-momentum tensor since only the commutator of the energy-momentum tensor appears.

(C) Similarly one can show that

$$
\frac{\delta}{\delta \boldsymbol{g}_{\mu \nu}(x)} P_{\boldsymbol{g}}=-\frac{i}{2}\left[T^{\mu \nu}(x), P\right]
$$

holds in the sense of quadratic forms on $\mathcal{V}_{\omega}$ for all polynomials

$$
P=\sum_{j \leq \ell, k_{j} \leq n} \check{\Phi}_{\omega}\left(\varphi_{j, 1}\right) \cdots \check{\Phi}_{\omega}\left(\varphi_{j, k_{j}}\right)
$$

in the field operators, with

$$
P_{\boldsymbol{g}}=\sum_{j \leq \ell, k_{j} \leq n} \check{\Phi}_{\omega}\left(F_{\boldsymbol{g}} \varphi_{j, 1}\right) \cdots \check{\Phi}_{\omega}\left(F_{\boldsymbol{g}} \varphi_{j, k_{j}}\right) .
$$

Proof. We will give the proof only for the case $\xi=0$ in order to simplify notation; however, the case of arbitrary $\xi$ can be carried out along the same lines. For any smooth curve $[0,1] \ni s \mapsto \boldsymbol{g}_{s} \in G$ with $\boldsymbol{g}_{s=0}=\stackrel{\circ}{\boldsymbol{g}}$, we will write $\delta \boldsymbol{g}=d \boldsymbol{g}_{s} /\left.d s\right|_{s=0}$, and $\delta y_{\boldsymbol{g}}=\left.\frac{d}{d s}\right|_{s=0} y_{\boldsymbol{g}_{s}}$ for any function $y_{\boldsymbol{g}}$ depending on $\boldsymbol{g} \in G$.

Let $\theta \in \mathcal{V}_{\omega}$. Since $\beta_{\boldsymbol{g}}(\stackrel{\circ}{W}(\varphi))=\stackrel{\circ}{W}\left(F_{\boldsymbol{g}} \varphi\right)$, one finds by a general argument (cf. e.g. 15]) that

$$
\delta\left(\beta_{\boldsymbol{g}} W\right)_{\omega} \theta=\delta\left(W\left(F_{\boldsymbol{g}} \varphi\right)\right)_{\omega} \theta=\frac{i}{2}\left\{\check{\Phi}_{\omega}\left(\delta F_{\boldsymbol{g}} \varphi\right), W_{\omega}(\varphi)\right\} \theta, \quad \varphi \in \stackrel{\circ}{\mathcal{R}},
$$

where $\{A, B\}=A B+B A$ denotes the anti-commutator. One must therefore derive an expression for $\delta F_{\boldsymbol{g}} \varphi$. It holds that (cf. (14))

$$
\begin{aligned}
\delta F_{\boldsymbol{g}} \varphi & =\delta\left(\stackrel{\circ}{E} K_{\boldsymbol{g}} \chi_{-}^{\mathrm{ret}} E_{\boldsymbol{g}} \stackrel{\circ}{K} \chi_{+}^{\mathrm{ret}} \varphi\right) \\
& =\stackrel{\circ}{E}\left(\delta K_{\boldsymbol{g}}\right) \chi_{-}^{\mathrm{ret}} \varphi+\stackrel{\circ}{E} \stackrel{\circ}{K} \chi_{-}^{\mathrm{ret}}\left(\delta E_{\boldsymbol{g}}\right) \chi_{+}^{\mathrm{ret}} \varphi .
\end{aligned}
$$

Now $\delta K_{\boldsymbol{g}}$ is a partial differential operator whose coefficient functions are compactly supported within $M_{(+,-)}$as a consequence of the geometric assumptions. Since $M_{(+,-)} \cap J^{-}\left(N_{-}\right)=\emptyset$, and $\operatorname{supp} \chi_{-}^{\text {ret }} \subset J^{-}\left(N_{-}\right)$, it follows that $\stackrel{\circ}{E}\left(\delta K_{\boldsymbol{g}} \chi_{-}^{\text {ret }}\right) \varphi=0$, and hence

$$
\delta F_{\boldsymbol{g}} \varphi=\stackrel{\circ}{E} \stackrel{\circ}{K} \chi_{-}^{\mathrm{ret}}\left(\delta E_{\boldsymbol{g}}\right) \stackrel{\circ}{K} \chi_{+}^{\mathrm{ret}} \varphi .
$$

On the other hand, it holds that

$$
\chi_{-}^{\mathrm{ret}} E_{\boldsymbol{g}} \stackrel{\circ}{K} \chi_{+}^{\mathrm{ret}} \varphi=\chi_{-}^{\mathrm{ret}} E_{\boldsymbol{g}}^{\mathrm{adv}} \stackrel{\circ}{K} \chi_{+}^{\mathrm{ret}} \varphi-\chi_{-}^{\mathrm{ret}} E_{\boldsymbol{g}}^{\mathrm{ret}} \stackrel{\circ}{K} \chi_{+}^{\mathrm{ret}} \varphi,
$$


and since $E_{\boldsymbol{g}}^{\text {adv }} \stackrel{\circ}{K} \chi_{+}^{\text {ret }}$ has support in $J^{+}\left(N_{+}\right)$, while $\chi_{-}^{\text {ret }}$ has support in $J^{-}\left(N_{-}\right)$, the first term on the right hand side vanishes, leaving us with

$$
\delta F_{\boldsymbol{g}} \varphi=-\stackrel{\circ}{E} \stackrel{\circ}{K} \chi_{-}^{\mathrm{ret}}\left(\delta E_{\boldsymbol{g}}^{\mathrm{ret}}\right) \stackrel{\circ}{K} \chi_{+}^{\mathrm{ret}} \varphi .
$$

Then we deduce from $E_{\boldsymbol{g}}^{\mathrm{ret}} K_{\boldsymbol{g}} f=f$ for all $f \in C_{0}^{\infty}(M, \mathbb{R})$ that

$$
\delta E_{\boldsymbol{g}}^{\mathrm{ret}}=-\stackrel{\circ}{E}^{\mathrm{ret}}\left(\delta K_{\boldsymbol{g}}\right) \stackrel{\circ}{E^{\mathrm{ret}}}
$$

and thus we obtain

$$
\delta F_{\boldsymbol{g}} \varphi=\stackrel{\circ}{E} \stackrel{\circ}{K} \chi_{-}^{\mathrm{ret}} \stackrel{\circ}{E^{\mathrm{ret}}}\left(\delta K_{\boldsymbol{g}}\right) \stackrel{\circ}{E^{\mathrm{ret}}} \stackrel{\circ}{K} \chi_{+}^{\mathrm{ret}} \varphi .
$$

Now we use the same support arguments as before to conclude that $\chi_{-}^{\text {ret }} E^{\text {adv }} \delta K_{\boldsymbol{g}}=$ 0 and $\delta K_{\boldsymbol{g}} \stackrel{\circ}{E}^{\text {adv }} \stackrel{\circ}{K} \chi_{+}^{\text {ret }} \varphi=0$, and hence it holds that

$$
\delta F_{\boldsymbol{g}} \varphi=\stackrel{\circ}{E} \stackrel{\circ}{K} \chi_{-}^{\mathrm{ret}} \stackrel{\circ}{E}\left(\delta K_{\boldsymbol{g}}\right) \stackrel{\circ}{E} \stackrel{\circ}{K} \chi_{+}^{\mathrm{ret}} \varphi=\stackrel{\circ}{E}\left(\delta K_{\boldsymbol{g}}\right) \varphi
$$

for all $\varphi \in \stackrel{\circ}{\mathcal{R}}$.

Therefore, our discussion so far shows that (15) is proved as soon as we have shown that, given $\boldsymbol{g} \in G$,

$$
\begin{aligned}
& \int\left\langle\theta,\left\{\Phi_{\omega}(x), W_{\omega}(\varphi)\right\} \theta\right\rangle\left(\delta K_{\boldsymbol{g}} \varphi\right)(x)(-\stackrel{\circ}{g})^{1 / 2}(x) d x \\
& =-\int\left\langle\theta,\left[T^{\mu \nu}(x), W_{\omega}(\varphi)\right] \theta\right\rangle \delta \boldsymbol{g}_{\mu \nu}(x)(-\stackrel{\circ}{g})^{1 / 2}(x) d x
\end{aligned}
$$

holds for all $\varphi \in \stackrel{\circ}{\mathcal{R}}$ and all $\theta \in \mathcal{V}_{\omega}$; note that $\delta K_{\boldsymbol{g}}$ is a differential operator on $C^{\infty}(M, \mathbb{R})$ containing $\delta \boldsymbol{g}_{\mu \nu}$. Due to the coordinate-independent nature of the integrals, it is sufficient to demonstrate that (16) holds in some arbitrarily chosen coordinate system for $M$. We may thus choose coordinates so that $-\stackrel{\circ}{g}(x)=1$ for all $x$. In such coordinates, one obtains

$$
\delta K_{\boldsymbol{g}}=\frac{1}{2} \stackrel{\circ}{\boldsymbol{g}}_{\mu \nu}\left(\partial^{\mu}\left(\stackrel{\circ}{\boldsymbol{g}}^{\alpha \beta} \delta \boldsymbol{g}_{\alpha \beta}\right)\right) \partial^{\nu}-\partial^{\mu} \delta \boldsymbol{g}_{\mu \nu} \partial^{\nu} \quad(-\stackrel{\circ}{g}=1) .
$$

Making also use of the fact that the $\delta \boldsymbol{g}_{\mu \nu}$ are compactly supported, and that $\varphi$ is a solution of the Klein-Gordon equation (1) on $(M, \stackrel{\circ}{g})$ (for $\xi=0)$, one obtains after partial integration in coordinates where $-\stackrel{\circ}{g}=1$,

$$
\begin{aligned}
& \text { 7) }\left\langle\theta,\left\{\Phi_{\omega}(x), W_{\omega}(\varphi)\right\} \theta\right\rangle\left(\delta K_{\boldsymbol{g}} \varphi\right)(x) d x \\
& =\int\left(\left\langle\theta,\left\{\partial^{\mu} \Phi_{\omega}(x), W_{\omega}(\varphi)\right\} \theta\right\rangle \partial^{\nu} \varphi(x)-\frac{1}{2} \stackrel{\circ}{g}^{\mu \nu}(x)\left\langle\theta,\left\{\partial^{\alpha} \Phi_{\omega}(x), W_{\omega}(\varphi)\right\} \theta\right\rangle \partial_{\alpha} \varphi(x)\right. \\
& \left.\quad+\frac{1}{2} \stackrel{\circ}{g}^{\mu \nu}(x) m^{2}\left\langle\theta,\left\{\Phi_{\omega}(x), W_{\omega}(\varphi)\right\} \theta\right\rangle \varphi(x)\right) \delta \boldsymbol{g}_{\mu \nu}(x) d x
\end{aligned}
$$

We shall next investigate the right hand side of (15)). The commutator of $W_{\omega}(\varphi)$ with the formal, point-split expression for the bitensor $T^{\mu \nu}\left(x, x^{\prime}\right)$ is given by

$$
\begin{aligned}
& \left\langle\theta,\left[T^{\mu \nu}\left(x, x^{\prime}\right), W_{\omega}(\varphi)\right] \theta\right\rangle=\left\langle\theta,\left[\partial^{\mu} \Phi_{\omega}(x) \partial^{\nu} \Phi_{\omega}\left(x^{\prime}\right), W_{\omega}(\varphi)\right] \theta\right\rangle \\
& -\frac{1}{2} \stackrel{\circ}{g}^{\mu \rho}(x) Y_{\rho}{ }^{\nu}\left(x, x^{\prime}\right)\left\langle\theta,\left[\left(\partial_{\alpha} \Phi_{\omega}(x) Y^{\alpha}{ }_{\beta}\left(x, x^{\prime}\right) \partial^{\beta} \Phi_{\omega}\left(x^{\prime}\right)-m^{2} \Phi_{\omega}(x) \Phi_{\omega}\left(x^{\prime}\right)\right), W_{\omega}(\varphi)\right] \theta\right\rangle
\end{aligned}
$$

where $Y^{\nu}{ }_{\alpha}\left(x, x^{\prime}\right)$ denotes the bitensor of parallel transport of vectors in $T_{x^{\prime}} M$ to $T_{x} M$. In order to be able to take the limit $x^{\prime} \rightarrow x$, one uses the relations

$$
\begin{gathered}
{\left[\Phi_{\omega}(h), W_{\omega}(\varphi)\right]=i\left[\Phi_{\omega}(h), \check{\Phi}_{\omega}(\varphi)\right] W_{\omega}(\varphi) \quad \text { and }} \\
i\left[\Phi_{\omega}(x), \check{\Phi}_{\omega}(\varphi)\right]=-\varphi(x), \quad h \in C_{0}^{\infty}(M, \mathbb{R}), \varphi \in \stackrel{\circ}{\mathcal{R}} ;
\end{gathered}
$$


the first relation holds generally in quasifree representations of the CCR-algebra as a consequence of the Weyl-relations, and the second relation is easily deduced from the equations

$$
\begin{gathered}
{\left[\Phi_{\omega}(h), \check{\Phi}_{\omega}(\varphi)\right]=i \sigma(E h, \varphi)=i \int h \varphi(-\stackrel{\circ}{g})^{1 / 2} d x,} \\
\left\langle\theta,\left[\Phi_{\omega}(h), \check{\Phi}_{\omega}(\varphi)\right] \theta\right\rangle=\int\left\langle\theta,\left[\Phi_{\omega}(x), \check{\Phi}_{\omega}(\varphi)\right] \theta\right\rangle h(x)(-\stackrel{\circ}{g})^{1 / 2}(x) d x
\end{gathered}
$$

which hold for all $h \in C_{0}^{\infty}(M, \mathbb{R}), \theta \in \mathcal{V}_{\omega}$. Inserting these relations together with the identity $[A B, C]=[A, C] B+A[B, C]$ yields for all $\theta \in \mathcal{V}_{\omega}$

$$
\begin{aligned}
\left\langle\theta,\left[T^{\mu \nu}\left(x, x^{\prime}\right), W_{\omega}(\varphi)\right] \theta\right\rangle & \\
= & -\left\langle\theta,\left(\partial^{\mu} \Phi_{\omega}(x) W_{\omega}(\varphi) \partial^{\nu} \varphi\left(x^{\prime}\right)+\partial^{\mu} \varphi(x) W_{\omega}(\varphi) \partial^{\mu} \Phi_{\omega}\left(x^{\prime}\right)\right) \theta\right\rangle \\
& +\frac{1}{2} \stackrel{\circ}{g}^{\mu \rho}(x) Y_{\rho}^{\nu}\left\langle\theta, Y_{\beta}^{\alpha}\left(\partial_{\alpha} \Phi_{\omega}(x) W_{\omega}(\varphi) \partial^{\beta} \varphi\left(x^{\prime}\right)+\partial_{\alpha} \varphi(x) W_{\omega}(\varphi) \partial^{\beta} \Phi_{\omega}\left(x^{\prime}\right)\right) \theta\right\rangle \\
& -\frac{1}{2} \stackrel{\circ}{g}^{\mu \rho}(x) Y_{\rho}^{\nu} m^{2}\left\langle\theta,\left(\Phi_{\omega}(x) W_{\omega}(\varphi) \varphi\left(x^{\prime}\right)+\varphi(x) W_{\omega}(\varphi) \Phi_{\omega}\left(x^{\prime}\right)\right) \theta\right\rangle
\end{aligned}
$$

where we have abbreviated $Y_{\rho}^{\nu}\left(x, x^{\prime}\right)$ by $Y_{\rho}^{\nu}$, etc. In the last expressions, one can clearly take the limit $x^{\prime} \rightarrow x$ without occurrence of any divergencies to obtain, upon observing $\delta \boldsymbol{g}_{\mu \nu}=\delta \boldsymbol{g}_{\nu \mu}$,

$$
\begin{aligned}
\left\langle\theta,\left[T^{\mu \nu}(x), W_{\omega}(\varphi)\right] \theta\right\rangle \delta \boldsymbol{g}_{\mu \nu}(x) & \\
= & -\left\langle\theta,\left\{\partial^{\mu} \Phi_{\omega}(x), W_{\omega}(\varphi)\right\} \theta\right\rangle \partial^{\nu} \varphi(x) \delta \boldsymbol{g}_{\mu \nu}(x) \\
& +\frac{1}{2} \stackrel{\circ}{g}^{\mu \nu}(x)\left\langle\theta,\left\{\partial_{\alpha} \Phi_{\omega}(x), W_{\omega}(\varphi)\right\} \theta\right\rangle \partial^{\alpha} \varphi(x) \delta \boldsymbol{g}_{\mu \nu}(x) \\
& -\frac{1}{2} \stackrel{\circ}{g}^{\mu \nu}(x) m^{2}\left\langle\theta,\left\{\Phi_{\omega}(x), W_{\omega}(\varphi)\right\} \theta\right\rangle \varphi(x) \delta \boldsymbol{g}_{\mu \nu}(x) .
\end{aligned}
$$

Comparing (17) and (18), one can see that the right hand side and the left hand side of (16) agree for coordinates where $-\stackrel{\circ}{g}=1$, for all $\varphi \in \stackrel{\circ}{\mathcal{R}}, \theta \in \mathcal{V}_{\omega}$, and all $\boldsymbol{g} \in G$. As this is sufficient for the validity of $(15)$, the proof is complete.

\section{WiCK-Polynomials}

The enlarged local algebras formed by the Wick polynomials defined in [7] (with the extended microlocal domain defined in [6]), which can be constructed in representations of quasifree Hadamard states of the free field over globally hyperbolic spacetimes, also satisfy the condition of local covariance. However, the Wickpolynomials themselves are in general not locally covariant quantum fields in the sense of Def. 2.4.

This point has been taken up recently by Hollands and Wald [24], and they have shown (among other things) that one may suitably define Wick-polynomials of the free scalar field which have the property to be locally covariant quantum fields in the sense of Def. 2.4. Here, we sketch an alternative -yet very much relatedapproach to constructing such locally covariant Wick-powers which emphasizes the cohomological nature of the problem.

Let, for $(M, \boldsymbol{g}) \in \mathrm{Obj}(\mathfrak{M a n}), \omega_{(M, \boldsymbol{g})}$ be a quasifree Hadamard state of the KleinGordon field on $(M, \boldsymbol{g})$, and let : $\Phi^{n}:_{\omega_{(M, g)}}$ denote the $n$-th Wick-power of the quantized Klein-Gordon field in the GNS-representation of $\omega=\omega_{(M, \boldsymbol{g})}$, as defined in [7, 6]. By $\mathscr{W}_{\omega}(M, \boldsymbol{g})$ we denote the unital $*$-algebra formed by all these Wick-powers. When $\omega^{\prime}=\omega_{(M, \boldsymbol{g})}^{\prime}$ is another quasifree Hadamard state, then one can show (cf. 24]) that there is a $*$-isomorphism $\hat{\alpha}: \mathscr{W}_{\omega}(M, \boldsymbol{g}) \rightarrow \mathscr{W}_{\omega^{\prime}}(M, \boldsymbol{g})$, so up to isomorphisms, $\mathscr{W}_{\omega}(M, \boldsymbol{g})$ is independent of the chosen quasifree Hadamard state. Now, to illustrate that the Wick-powers defined with respect to a reference Hadamard state are not locally covariant, and how they may be re-defined to become locally covariant in 
terms of the solution of a cohomological problem, we will consider in the following the case of the Wick-square.

In order that the family $\left\{: \Phi^{2}:_{\omega_{(M, g)}}\right\}$ of Wick-squares, defined with respect to a family $\left\{\omega_{(M, g)}\right\}$ of quasifree Hadamard states, be a locally covariant quantum field, it is required that there is for any $\psi \in \operatorname{hom}_{\mathfrak{M a n}}\left((M, \boldsymbol{g}),\left(M^{\prime}, \boldsymbol{g}^{\prime}\right)\right)$ a unital $*$-algebraic morphism $\gamma_{\psi}: \mathscr{W}_{\omega}(M, \boldsymbol{g}) \rightarrow \mathscr{W}_{\omega}\left(M^{\prime}, \boldsymbol{g}^{\prime}\right)$ so that $\gamma_{\psi}\left(: \Phi^{2}:_{\omega_{(M, g)}}\right)=: \Phi^{2}:_{\omega_{\left(M^{\prime}, \boldsymbol{g}^{\prime}\right)}} \circ \psi_{*}$. But actually it holds that

$\tilde{\alpha}_{\psi}\left(: \Phi^{2}: \omega_{(M, \boldsymbol{g})}(x)\right)=: \Phi^{2}: \omega_{\left(M^{\prime}, \boldsymbol{g}^{\prime}\right)}(\psi(x))+\left(w_{2}^{\left(\omega_{\left(M^{\prime}, \boldsymbol{g}^{\prime}\right)}\right)}(\psi(x), \psi(x))-w_{2}^{\left(\omega_{(M, \boldsymbol{g})}\right)}(x, x)\right)$

where we have indicated distributions by their variable entries $(x)$, and $\tilde{\alpha}_{\psi}$ is an appropriate extension of the $C^{*}$-algebraic morphism associated with the KleinGordon field's functor $\mathscr{A}$. Thus, the difference term on the left hand side will have to vanish in order that $\left\{: \Phi^{2}:_{(M, g)}\right\}$ be a locally covariant field. As we have already indicated in Sec. 3.1, this will not hold in general (see also [24]).

Let us indicate how this problem may be solved. If $\omega=\omega_{\left(M^{\prime}, \boldsymbol{g}^{\prime}\right)}$ and $\omega^{\prime}=\omega_{\left(M^{\prime}, \boldsymbol{g}^{\prime}\right)}$ are two quasifree Hadamard states over the spacetime $\left(M^{\prime}, \boldsymbol{g}^{\prime}\right)$, then there is a smooth function $B_{\omega, \omega^{\prime}}$ on $M^{\prime}$ so that : $\Phi^{2}: \omega\left(x^{\prime}\right)-: \Phi^{2}:_{\omega^{\prime}}\left(x^{\prime}\right)=B_{\omega, \omega^{\prime}}\left(x^{\prime}\right)$. These functions satisfy the covariance condition

$$
B_{\omega \circ \tilde{\alpha}_{\psi}, \omega^{\prime} \circ \tilde{\alpha}_{\psi}}(x)=B_{\omega, \omega^{\prime}}(\psi(x)), \quad x \in M,
$$

for $\psi \in \operatorname{hom}_{\mathfrak{M a n}}\left((M, \boldsymbol{g}),\left(M^{\prime}, \boldsymbol{g}^{\prime}\right)\right)$, and moreover, they fulfill a cocycle condition

$$
B_{\omega, \omega^{\prime}}+B_{\omega^{\prime}, \omega^{\prime \prime}}+B_{\omega^{\prime \prime}, \omega}=0 .
$$

The aim is now to trivialize this cocycle while preserving its covariance properties. In other words, we are seeking to associate with each quasifree Hadamard state $\omega=\omega_{\left(M^{\prime}, \boldsymbol{g}^{\prime}\right)}$ over $\left(M^{\prime}, \boldsymbol{g}^{\prime}\right)$ a smooth function $f_{\omega_{\left(M^{\prime}, g^{\prime}\right)}} \in C^{\infty}\left(M^{\prime}\right)$ so that the resulting family of smooth functions transforms covariantly, i.e.

$$
f_{\omega \circ \tilde{\alpha}_{\psi}}(x)=f_{\omega}(\psi(x)), \quad \psi \in \operatorname{hom}_{\mathfrak{M a n}}\left((M, \boldsymbol{g}),\left(M^{\prime}, \boldsymbol{g}^{\prime}\right)\right),
$$

and trivializes the cocycle, i.e.

$$
B_{\omega, \omega^{\prime}}\left(x^{\prime}\right)=f_{\omega}\left(x^{\prime}\right)-f_{\omega^{\prime}}\left(x^{\prime}\right), \quad x^{\prime} \in M^{\prime},
$$

for $\omega=\omega_{\left(M^{\prime}, \boldsymbol{g}^{\prime}\right)}, \omega^{\prime}=\omega_{\left(M^{\prime}, \boldsymbol{g}^{\prime}\right)}^{\prime}$ any pair of quasifree Hadamard states over $(M, \boldsymbol{g})$. Hence we would obtain a locally covariant Wick-square by setting

$$
: \Phi^{2}:_{(M, \boldsymbol{g})}(x)=: \Phi^{2}:_{\omega_{(M, \boldsymbol{g})}}(x)-f_{\omega_{(M, \boldsymbol{g})}}(x)
$$

for arbitrary choice a of quasifree Hadamard state $\omega_{(M, \boldsymbol{g})}$ over $(M, \boldsymbol{g})$.

It is not too difficult to find the solution to this cohomological problem. Recalling the definition of the Hadamard form by Kay and Wald [29], one finds that the diagonal values of the smooth, non-geometrical term $H_{\omega}$ (cf. (11)) of the two-point function of a quasifree Hadamard state $\omega$ have the required properties, i.e. a solution of the cohomological problem is provided by defining

$$
f_{\omega}(x)=H_{\omega}(x, x), \quad x \in M,
$$

for all quasifree Hadamard states $\omega=\omega_{(M, \boldsymbol{g})}$ over $(M, \boldsymbol{g})$. Actually, $H_{\omega}(x, y)$ is defined off the diagonal $x=y$ only up to a $C^{\infty}$-function owing to the fact that the geometrical terms $G_{\epsilon}$ are affected by the like ambiguity. However, one can show that this ambiguity vanishes for $y \rightarrow x$ and that, consequently, $H_{\omega}(x, x)$ is well-defined, see the discussion in Sec. 5.2 of 24].

Higher order Wick-powers which are also locally covariant may then by obtained by differentiating the generating functional

$$
: e^{\lambda \Phi(x)}:_{(M, g)}=e^{\frac{1}{2} \lambda^{2} f_{\omega}(x)}: e^{\lambda \Phi(x)}: \omega
$$


with respect to the real parameter $\lambda$, where $\omega=\omega_{(M, g)}$ is any quasifree Hadamard state over $(M, \boldsymbol{g})$.

Finally we remark that we have only considered Wick-powers without derivatives. A discussion of Wick-powers with derivatives is contained in a recent work by Moretti [31].

Acknowledgments. We would like to thank Stefan Hollands, Bernard Kay and Robert Wald for discussions which were stimulating for the development of the present work.

\section{APPENDix}

a) Proof of statement $(\alpha)$ in the proof of Thm. 3.9

It is clearly sufficient to prove that $\boldsymbol{F}\left(\pi_{\omega \circ \beta}\right) \subset \boldsymbol{F}\left(\pi_{\omega} \circ \beta\right)$ for all states $\omega$ on a $C^{*}$-algebra $\mathcal{B}$ and all $C^{*}$-algebraic endomorphisms $\beta: \mathcal{A} \rightarrow \mathcal{B}$, where $\mathcal{A}$ is another $C^{*}$-algebra. Consider the GNS-representation $\left(\mathcal{H}_{\omega}, \pi_{\omega}, \Omega_{\omega}\right)$ of $\mathcal{B}$ corresponding to the state $\omega$. Define a new Hilbert-space $\mathcal{H}^{\alpha}$ as the closed subspace of $\mathcal{H}_{\omega}$ which is spanned by $\pi_{\omega}(\alpha(\mathcal{A})) \Omega_{\omega}$. Then we may clearly identify the GNS-representation $\left(\mathcal{H}_{\omega \circ \alpha}, \pi_{\omega \circ \alpha}, \Omega_{\omega \circ \alpha}\right)$ of $\mathcal{A}$ induced by the state $\omega \circ \alpha$ with $\left(\mathcal{H}^{\alpha}, \pi_{\omega} \circ \alpha, \Omega_{\omega}\right)$ since this triple has all the properties of the GNS-triple corresponding to $\omega \circ \alpha$, and the GNS-triple is unique (up to unitary identifications). Hence, if $\omega^{\prime} \in \boldsymbol{F}\left(\pi_{\omega \circ \alpha}\right)$, then there is a density matrix $\rho^{\prime}=\sum_{j} \mu_{j}\left|\phi_{j}\right\rangle\left\langle\phi_{j}\right|$ with unit vectors $\phi_{j} \in \mathcal{H}^{\alpha}$ such that

$$
\omega^{\prime}(A)=\operatorname{tr}\left(\rho^{\prime} \pi_{\omega} \circ \alpha(A)\right)
$$

holds for all $A \in \mathcal{A}$. This density matrix is then also a density matrix on $\mathcal{H}_{\omega} \supset \mathcal{H}^{\alpha}$, and owing to the just displayed equality, then also $\omega^{\prime} \in \boldsymbol{F}\left(\pi_{\omega} \circ \alpha\right)$ according to the definition of the folium of a representation.

\section{b) Proof of statement $(\beta)$ in the proof of Thm. 3.2}

We quote the following result which is proved as Prop. 5.3.5 in 12: Let $\mathcal{B}$ be a $C^{*}$-algebra and $\pi$ a representation of $\mathcal{B}$ on some Hilbert-space $\mathcal{H} ;$ moreover, let $\mathcal{H}^{\prime}$ be a closed subspace of $\mathcal{H}$ which is left invariant by $\pi(\mathcal{B})$ and non-zero, and define the subrepresentation $\pi^{\prime}(B)=\pi(B) \uparrow \mathcal{H}^{\prime}, B \in \mathcal{B}$, of $\pi$ on $\mathcal{H}^{\prime}$. Then $\pi$ is quasi-equivalent to $\pi^{\prime}$ if the von Neumann algebra $\pi(\mathcal{B})^{\prime \prime}$ is a factor.

We apply this to prove statement $(\beta)$ as follows: Let $\pi$ the identical representation of the factor $\mathcal{N}$ on the Hilbert-space $\mathcal{H}$, and let $\pi^{\prime}$ the subrepresentation relative to $\mathcal{H}^{\prime}=\mathcal{H}_{\mathcal{N}}$. According to the quoted result, $\boldsymbol{F}(\pi)=\boldsymbol{F}\left(\pi^{\prime}\right)$. And this just says that for each density matrix $\rho$ on $\mathcal{H}$ there exists a density matrix $\rho^{\mathcal{N}}$ on $\mathcal{H}_{\mathcal{H}}=\mathcal{H}^{\prime}$ so that

$$
\operatorname{tr}(\rho \cdot N)=\operatorname{tr}(\rho \cdot \pi(N))=\operatorname{tr}\left(\rho^{\mathcal{N}} \cdot \pi^{\prime}(N)\right)=\operatorname{tr}\left(\rho^{\mathcal{N}} \cdot N\right)
$$

holds for all $N \in \mathcal{N}$.

\section{REFERENCES}

[1] Araki, H., Mathematical theory of quantum fields, Oxford University Press, Oxford, 1999

[2] Atiyah, M., "Topological quantum field theories", Publ. Math. IHES Paris 68, 178 (1985)

[3] Bannier, U., "On generally covariant quantum field theory and generalized causal and dynamical structures", Commun. Math. Phys. 118, 163 (1988)

[4] Borchers, H.J., "On the structure of the algebra of field operators", Nuovo Cimento 24, 214 (1962)

[5] Bratteli, O., Robinson, D.W., Operator algebras and quantum statistical mechanics, vol. 1, 2nd edn., Springer-Verlag, Berlin, 1987

[6] Brunetti, R., Fredenhagen, K., "Microlocal analysis and interacting quantum field theories: Renormalization on physical backgrounds", Commun. Math. Phys. 208, 623 (2000) 
[7] Brunetti, R., Fredenhagen, K., Köhler, M., "The microlocal spectrum condition and Wick polynomials on curved spacetime", Commun. Math. Phys. 180, 633 (1996)

[8] Buchholz, D., "Product states for local algebras", Commun. Math. Phys. 36, 287 (1974)

[9] Buchholz, D., Wichmann, E.H., "Causal independence and the energy-level density of states in quantum field theory", Commun. Math. Phys. 106, 321 (1986)

[10] Dimock, J., "Algebras of local observables on a manifold", Commun. Math. Phys. 77, 219 (1980)

[11] Dimock, J., "Dirac quantum fields on a manifold", Trans. Amer. Math. Soc. 269, 133 (1982)

[12] Dixmier, J., Les $C^{*}$-algèbres et leurs représentations, Gauthier-Villars, Paris, 1964

[13] Dosch, H.G., Müller, V.F., "Renormalization of quantum electrodynamics in an arbitrarily strong time-independent external field", Fortsch. Phys. 23, 661 (1975)

[14] Dyson, F.J., "Missed opportunities", Bull. Am. Math. Soc. 78, 635 (1972)

[15] Fewster, C.J., Verch, R., "Passivity from quantum weak energy inequalities", in preparation.

[16] Fredenhagen, K., Haag, R., "Generally covariant quantum field theory and scaling limits", Commun. Math. Phys. 108, 91 (1987)

[17] Fulling, S.A., "Non-uniqueness of canonical field quantization in Riemannian space-time", Phys. Rev. D 7, 2850 (1973)

[18] Fulling, S.A., Narcowich, F.J., Wald, R.M., "Singularity structure of the two-point function in quantum field theory in curved spacetime, II", Ann. Phys. (N.Y.) 136, 243 (1981)

[19] Haag, R., Local Quantum Physics, 2nd ed. Springer-Verlag, Berlin, Heidelberg, New York, 1996

[20] Haag, R., Narnhofer, H., Stein, U., "On quantum field theories in gravitational background", Commun. Math. Phys. 94, 219 (1984)

[21] Haag, R., Kastler, D., "An algebraic approach to quantum field theory", J. Math. Phys. 5, 848 (1964)

[22] Hawking, S.W., "Particle creation by black holes", Commun. Math. Phys. 43, 199 (1975)

[23] Hawking, S.W., Ellis, G.F.R., The large scale structure of space-time, Cambridge University Press, Cambridge, 1973

[24] Hollands, S., Wald, R.M., "Local Wick polynomials and time ordered products of quantum fields in curved spacetime", Commun. Math. Phys. 223, 289 (2001)

[25] Hollands, S., Wald, R.M., "Existence of local covariant time orederd products of quantum fields in curved spacetime", preprint arXiv:gr-qc/0111108

[26] Hörmander, L., The analysis of partial differential operators, Vol 1, Springer-Verlag, BerlinHeidelberg-New York, 1983

[27] Kay, B.S., "Quantum fields in curved spacetime: Non global hyperbolicity and locality", in the Proceedings of the Conference Operator algebras and quantum field theory held in Rome, July 1996, S. Doplicher, R. Longo, J.E. Roberts, L. Zsido eds, International Press, 1997

[28] Kay, B.S., "Linear spin-0 quantum fields in external gravitational and scalar fields, II," Commun. Math. Phys. 71, 29 (1980)

[29] Kay, B.S., Wald, R.M., "Theorems on the uniqueness and thermal properties of stationary, nonsingular, quasifree states on spacetimes with a bifurcate Killing horizon", Phys. Rep. 207, 49 (1991)

[30] Mac Lane, S., Categories for the working mathematician, Springer-Verlag, New York, 1971

[31] Moretti. V.. "Comments on the stress-energy operator in curved spacetime", preprint arXiv:gr-qc/0109048

[32] Radzikowski, M.J., "Micro-local approach to the Hadamard condition in quantum field theory in curved spacetime", Commun. Math. Phys. 179, 529 (1996)

[33] Sahlmann, H., Verch. R., "Passivity and microlocal spectrum condition", Commun. Math. Phys. 214, 705 (2000)

[34] Sahlmann, H., Verch, R., "Microlocal spectrum condition and Hadamard form for vectorvalued quantum fields in curved spacetime", Rev. Math. Phys. 13, 1203 (2001)

[35] Summers, S.J., "Normal product states for fermions and twisted duality for CCR- and CARtype algebras with applications to the Yukawa 2 quantum field model", Commun. Math. Phys. 86, $111(1982)$

[36] Summers, S.J., "On the independence of local algebras in quantum field theory", Rev. Math. Phys. 2, 201 (1990)

[37] Torre, C.G., Varadarajan, M., "Functional evolution of free quantum fields", Class. Quantum Grav. 16, 2651 (1999)

[38] Uhlmann, A., "Über die Definition der Quantenfelder nach Wightman und Haag", Wiss. Zeits. Karl Marx Univ. Leipzig 11, 213 (1962)

[39] Verch, R., "Local definiteness, primarity and quasiequivalence of quasifree Hadamard quantum states in curved spacetime", Commun. Math. Phys. 160, 507 (1994) 
[40] Verch, R., "Continuity of symplectically adjoint maps and the algebraic structure of Hadamard vacuum representations for quantum fields on curved spacetime", Rev. Math. Phys. 9, 635 (1997)

[41] Verch, R., "Wavefront sets in algebraic quantum field theory", Commun. Math. Phys. 205, $337(1999)$

[42] Verch, R., "A spin-statistics theorem for quantum fields on curved spacetime manifolds in a generally covariant framework", Commun. Math. Phys. 223, 261 (2001)

[43] Unruh, W.G., "Notes on black hole evaporation", Phys. Rev. D 14, 870 (1976)

[44] Wald, R.M., "The back reaction effect in particle creation in curved spacetime" Commun. Math. Phys. 54, 1 (1977)

[45] Wald, R.M., "Existence of the $S$-matrix in quantum field theory in curved spacetime", Ann. Phys. (N.Y.) 118, 490 (1979)

[46] Wald, R.M., General relativity, University of Chicago Press, Chicago, 1984

[47] Wald, R.M., Quantum field theory in curved spacetime and black hole thermodynamics, University of Chicago Press, Chicago, 1994 